\title{
Role of synaptic nonlinearity in persistent firing rate shifts caused by external periodic forcing
}

\author{
Nikita Novikov $\odot^{1, *}$ and Boris Gutkin ${ }^{1,2}$ \\ ${ }^{1}$ Centre for Cognition and Decision Making, National Research University Higher School of Economics, Moscow 101000, Russia \\ ${ }^{2}$ Group for Neural Theory, LNC INSERM U960, Department of Cognitive Studies, Ecole Normale Superieure PSL Research University, \\ Paris 75005 , France
}

(Received 27 February 2019; revised manuscript received 21 October 2019; accepted 3 March 2020; published 21 May 2020)

\begin{abstract}
Information storage and processing in the brain largely relies on the neural population coding principle. In this framework, information is reflected in the population firing rate that reflects asynchronous irregular spiking of its constituent neurons. Periodic modulations of neural activity can lead to neural activity oscillations. Data indicate that such oscillations are ubiquitous in brain activity and are modulated, in frequency and amplitude, in a functionally meaningful manner. The relationship between oscillations and the population rate code remains an open issue. While ample works show how changes in the mean firing rate may alter neural oscillations, the reverse connection is unclear. One notable possibility is that oscillatory activity impinging on a neural population modulates its mean firing rate, thereby impacting information processing. We suggest that such modulation requires nonlinearities and propose nonlinear excitatory coupling via slow N-methyl-D-aspartate (NMDA) receptors as the prevalent mechanism. The aim of our paper is to theoretically explore to what extent the NMDA-related mechanism could account for oscillation-induced mean firing rate changes. We consider a mean-field model of a neural circuit containing an excitatory and an inhibitory population with linear transfer functions. Along with NMDA excitation, the model included fast recurrent excitatory and inhibitory connectivity. To explicitly study the effects of impinging oscillation on the rate dynamics, we subjected the circuit to a sinusoidal input signal imitating an input from distant brain regions or from a larger network into which the circuit is embedded. Using time-scale separation and time-averaging techniques, we developed a geometric method to determine the oscillation-induced mean firing rate shifts and validated it by numeric simulations of the model. Our results indicate that a large-amplitude stable firing rate shift requires nonlinear NMDA synapses on both the excitatory and the inhibitory populations. Our results delineate specific neural synaptic properties that enable neural oscillations to act as flexible modulators of the population rate code.
\end{abstract}

DOI: 10.1103/PhysRevE.101.052408

\section{INTRODUCTION}

Neurons in cortical circuits tend to generate irregular spike trains with low correlation between individual neurons. In this regime, the overall activity of a homogenous neural population can be described by the population firing rate- the total number of spikes generated by neurons of this population per unit time. In many cases, the population firing rate is periodically modulated in time-a phenomenon known as neural oscillations. Data show that such oscillations are abundant across brain networks, and their characteristics are sensitive to the various experimental and natural conditions [1]. This oscillatory activity is essentially a collective phenomenon (as opposed to the individual spike trains generated by the individual neurons). Properties of the population oscillations are related to the mean population firing rate, but this relation

\footnotetext{
*nikknovikov@gmail.com
}

Published by the American Physical Society under the terms of the Creative Commons Attribution 4.0 International license. Further distribution of this work must maintain attribution to the author(s) and the published article's title, journal citation, and DOI. is not straightforward; for example, the oscillation frequency could be very different from the firing rate. Experimental evidence also suggests that oscillatory properties could be modulated independently of firing rates [2].

One of the most prominent principles of information encoding in the brain is the population (rate) coding [3] framework. In this scheme, firing rates of stimulus-tuned populations are the main information carrier in neural activity. For example, if a neural population consistently increases or decreases its firing rate when perceived or internally generated information contains a certain feature, it is said to be selective for this feature. Considering the firing rates of many (potentially overlapping) neural populations, one could decode complex multidimensional information.

While rate coding has been amply explored, the role of oscillations in encoding and routing of information in the brain is much less understood. Notably, the interplay between the rate coding and the oscillatory activity remains a largely open question. In the literature, there have been several proposals on how oscillatory activity could be related to rate coding. In the first scenario, either frequency or amplitude of oscillations carries additional information not contained in the firing rates [4]. In the second scenario, information is still encoded in the mean population firing rates, but the 
way this information would propagate in the brain depends on the oscillatory activity of these populations [5,6]. In the third scenario, oscillations themselves do not carry information, but instead modulate properties of the existing firing rate code (e.g., make it more robust) [7,8,9].

In order for any of these scenarios to work, there should be neural mechanisms of transformations between mean population firing rate and oscillatory properties. [5,10]. Mechanisms by which the mean firing rate could affect oscillatory properties are quite well understood $[11,12,13]$. For example, if a population increases its firing rate after receiving a signal for which it is selective, it could transition from the stationary state (with constant firing rate) to self-generated oscillations [11]. At the same time, the mechanisms by which the oscillations influence the mean population firing rate are much less studied. Let us consider a neuronal network system that does not generate oscillations by itself, but rather receives an external zero-mean sinusoidal input that can be varied independently of the system's parameters (by "external" we mention an input that comes from some unspecified brain structure that is not directly included in the system under consideration). In this case, we can focus on the internal properties of the system that account for oscillation-induced changes of the mean population firing rate.

First of all, in order for zero-mean sinusoidal input to control (modify) the mean firing rate, the system should be nonlinear. The most obvious candidate property that could mediate oscillatory control of the mean firing rate is nonlinearity of individual neurons' gain functions (i.e., relations between the input strength and the output firing rate) $[2,14,15]$. If the baseline firing rate is low, this mechanism could potentially play a significant role (as the firing rate is strictly positive, so the nonlinearity is strong in this case). However, in a high firing rate regime with irregular spiking activity (e.g., typical for working memory retention), the gain functions are close to linear, so the aforementioned effect should be quite subtle.

A further possibility to make zero-mean oscillations affect the population firing rate is to implement a network-wide nonlinearity by introducing recurrent excitatory synaptic coupling mediated by N-methyl-D-aspartate (NMDA) receptors. Biophysically speaking, the NMDA-mediated synaptic current depends on the presynaptic firing rate (which controls neurotransmitter release), as well as on postsynaptic cell membrane voltage (which controls release of NMDA receptors from a magnesium block). Since both of these quantities, the firing rate and the membrane voltage, reflect the level of the network activity, NMDA currents depend on this activity level in a highly nonlinear manner, which makes it a good candidate for providing the oscillatory-induced mean firing rate modulation.

In the present paper, we consider a neuronal network containing one excitatory and one inhibitory population with external zero-mean sinusoidal forcing. We theoretically investigate the role of nonlinear NMDA currents in mediating excitatory influences of the periodic forcing on the mean firing rates of the system. To render the system analyzable, we use a macroscopic low-dimensional model, the variables of which represent the population firing rates and the mean NMDA currents. We separate the effects of the NMDA and of the gain function nonlinearities, by considering a model with linearized neuronal gain functions. We demonstrate that in the presence of NMDA-containing synapses on the excitatory population only oscillatory input could induce only a small positive shift of the firing rate $(1-2 \mathrm{~Hz})$ before loss of stability. However, when NMDA-mediated excitation was also present on the inhibitory population, large positive stable shifts $(10-20 \mathrm{~Hz})$ of excitatory firing rates could be achieved. Thereby we show that synaptic nonlinearities on both the excitatory and the inhibitory neuronal populations are crucial for significant interactions between oscillatory inputs and the mean population firing rate.

The paper is organized as follows.

(1) We describe our model and explore analytically the relationship between the oscillatory forcing parameters and the mean firing rate shift.

(2) We perform a phase plane analysis for the system with NMDA receptors located on the excitatory population only and discuss the resulting limitations on the mean firing rate shift. We confirm our predictions by numerical simulation of the model.

(3) We perform a phase plane analysis and confirming simulation for the system with NMDA receptors located on both populations (the excitatory and the inhibitory). We discuss how adding NMDA receptors to the inhibitory population helps to overcome the limitations on the mean firing rate shift.

(4) We systematically explore the parameter space and discuss which parameter combinations allow one to achieve the highest values of the mean firing rate shift.

\section{THEORY}

\section{A. The low-dimensional neural circuit model}

We study neural population firing rate dynamics of an excitatory-inhibitory neural circuit where the two different neural populations are recurrently intercoupled with instantaneous excitatory alpha-amino-3-hydroxy-5-methyl-4isoxazole propionic acid (AMPA) and inhibitory gammaaminobutyric acid receptor type A, as well as slow nonlinear excitatory NMDA, connections. Both the excitatory and the inhibitory populations receive external tonic input, as well as nonzero sinusoidal signal (both the tonic and the sinusoidal inputs are assumed to come from unspecified parts of the brain that are not directly included in our system). The network dynamics are given by a system of first-order differential equations:

$$
\begin{aligned}
\frac{d r_{e}}{d t} & =\frac{1}{\tau_{r e}}\left(r_{e}^{s s, 0}\left(u_{e}\left(r_{e}, r_{i}, I_{\mathrm{NMDA} e}\right)\right)-r_{e}\right) \\
\frac{d r_{i}}{d t} & =\frac{1}{\tau_{r i}}\left(r_{i}^{s s, 0}\left(u_{i}\left(r_{e}, r_{i}, I_{\mathrm{NMDA} i}\right)\right)-r_{i}\right) \\
\frac{d V_{e}}{d t} & =\frac{1}{\tau_{V e}}\left(V_{e}^{s s, 0}\left(u_{e}\left(r_{e}, r_{i}, I_{\mathrm{NMDA} e}\right)\right)-V_{e}\right) \\
\frac{d V_{i}}{d t} & =\frac{1}{\tau_{V i}}\left(V_{i}^{s s, 0}\left(u_{i}\left(r_{e}, r_{i}, I_{\mathrm{NMDA} i}\right)\right)-V_{i}\right) \\
\frac{d I_{\mathrm{NMDA} e}}{d t} & =\frac{1}{\tau_{\mathrm{NMDA}}}\left(I_{\mathrm{NMDA} e}^{s s, 0}\left(r_{e}, V_{e}\right)-I_{\mathrm{NMDA} e}\right) \\
\frac{d I_{\mathrm{NMDA} i}}{d t} & =\frac{1}{\tau_{\mathrm{NMDA}}}\left(I_{\mathrm{NMDA} i}^{s s, 0}\left(r_{e}, V_{i}\right)-I_{\mathrm{NMDA} i}\right),
\end{aligned}
$$


where $r_{e}, r_{i}$ are the excitatory and inhibitory population firing rates (i.e., spikes per second, given in units of $\mathrm{Hz}) ; V_{e}, V_{i}$ are the mean population membrane voltages; $I_{\mathrm{NMDA} e}, I_{\mathrm{NMDA} i}$ are the mean NMDA currents received by the populations; time scales are defined by the time constants $\tau_{r e}, \tau_{r i}, \tau_{V e}, \tau_{V i}, \tau_{\mathrm{NMDA}} ; u_{e}, u_{i}$ denote the mean instantaneous inputs to the excitatory and the inhibitory populations, respectively (expressed in terms of voltage). The functions $r_{e}^{s s, 0}\left(u_{e}\right), r_{i}^{s s, 0}\left(u_{i}\right), V_{e}^{s s, 0}\left(u_{e}\right), V_{i}^{s s, 0}\left(u_{i}\right)$ give the state-dependent instantaneous equilibrium values; we derive these functions from simulations of individual neurons (see Appendix A2). The effect of the tonic input noise is incorporated in the shapes of these functions, and the system (1) itself is deterministic. The functions $I_{\mathrm{NMDAe}}^{s s, 0}\left(r_{e}, V_{e}\right), I_{\mathrm{NMDA} i}^{s s, 0}\left(r_{e}, V_{i}\right)$ giving the nonlinear NMDA currents take the presynaptic excitatory firing rate and the mean postsynaptic voltage as arguments and are expressed as follows:

$$
I_{\mathrm{NMDA} a}^{s s, 0}\left(r_{e}, V_{a}\right)=J_{a e}^{\mathrm{NMDA}} g_{\mathrm{NMDA}}\left(V_{a}\right) r_{e}
$$

where the index $a$ could be replaced by $e$ for the excitatory population or by $i$ for the inhibitory population (this notation will be used throughout the paper). Here $J_{a e}^{\mathrm{NMDA}}$ is the strength of the NMDA-related coupling between the excitatory population and the population $a$, in units of (As). The function $g_{\text {NMDA }}$ gives a nondimensional coefficient that describes the dependence of NMDA current on the membrane voltage:

$$
g_{\mathrm{NMDA}}(V)=\left[1+0.28 \exp \left(-\frac{V}{V_{\mathrm{NMDA}}}\right)\right]^{-1},
$$

where $V_{\mathrm{NMDA}}=0.016(\mathrm{~V})$.

The inputs $u_{e}, u_{i}$ depend on the firing rates $r_{e}, r_{i}$, the external tonic inputs $h_{e}, h_{i}$ (expressed in voltage units), and the NMDA currents $I_{\mathrm{NMDA} e}, I_{\mathrm{NMDA} i}$ as follows:

$$
u_{a}\left(r_{e}, r_{i}, I_{\mathrm{NMDA} a}\right)=J_{a e}^{\mathrm{AMPA}} r_{e}-J_{a i} r_{i}+h_{a}+I_{\mathrm{NMDA} a} / g_{m a},
$$

where $J_{a e}^{\mathrm{AMPA}}, J_{a i}$ are the strengths of the fast synaptic connections (in units of $(\mathrm{Vs})$; the first index defines the input population, and the second index defines the output population), and $g_{m a}$ is the membrane conductance per unit area of neurons from the population $a$. The synaptic coupling strengths could be expressed as follows:

$$
\begin{aligned}
J_{a e}^{\mathrm{AMPA}} & =j_{a e}^{\mathrm{AMPA}} K_{a e} \tau_{m a} \\
J_{a e}^{\mathrm{NMDA}} & =j_{a e}^{\mathrm{NMDA}} K_{a e} \tau_{\mathrm{NMDA}} \\
J_{a i} & =j_{a i} K_{a i} \tau_{m a},
\end{aligned}
$$

where $j_{a e}^{\mathrm{AMPA}}$ and $j_{a i}$ are the amplitudes of the instantaneous excitatory and inhibitory postsynaptic potentials on the neurons from the population $a$ (given in voltage units); $j_{a e}^{\mathrm{NMDA}}$ is the amplitude of the NMDA-postsynaptic current step onto the neurons from the population $a$ (given in current units); $K_{a b}$ is number of inputs that a neuron from population $a$ receives from neurons that belong to a population $b(a, b=e, i)$; $\tau_{m a}$ is the membrane time constant of the neurons from the population $a$.

The time constant of the NMDA-synaptic input is much larger than the time constants of the population firing rate and the membrane voltage dynamics. Thus, we can consider our system as a slow-fast system, and separate the time scales. At the fast time scale, the state of the slow subsystem $z_{s} \equiv\left(I_{\mathrm{NMDA} e}, I_{\mathrm{NMDA} i}\right)$ can be considered as constant; then the state of the fast subsystem $z_{F} \equiv\left(r_{e}, r_{i}, V_{e}, V_{i}\right)$ converges to an equilibrium $z_{F}^{*} \equiv\left(r_{e}^{*}, r_{i}^{*}, V_{e}^{*}, V_{i}^{*}\right)$ that depends on $z_{s}$. At the slow time scale, the slow subsystem evolves, and its dynamics assume that the fast subsystem is converged to its instantaneous equilibrium at each time moment, i.e., $z_{F}=z_{F}^{*}$. Further in the paper, we use the time scale separation to analyze stability of the fast and the slow subsystems independently, as well as to analyze the effect of forced oscillations of the fast subsystem on the dynamics of the slow subsystem.

\section{B. Characteristic curves and steady states of the linearized system}

The impact of zero-mean periodic forcing on the average firing rate could be mediated by nonlinearity of the fast subsystem (given by the functions $r_{a}^{s s, 0}, V_{a}^{s s, 0}$ ), as well as the NMDA nonlinearity (given by the functions $\left.I_{\mathrm{NMDA} a}^{s s, 0}\right)$. In this paper, we concentrate on the effect of the $I_{\mathrm{NMDA} a}^{S s, 0}$ nonlinearity, so to analyze it separately we linearize $r_{a}^{s s, 0}, V_{a}^{s s, 0}$ about the steady state. We denote this state as $S_{0} \equiv\left(r_{e 0}, r_{i 0}, V_{e 0}, V_{i 0}, I_{\mathrm{NMDA} e 0}, I_{\mathrm{NMDA} i 0}\right)$, and the corresponding inputs as $\left(u_{e 0}, u_{i 0}\right)$. The linearized system is given by

$$
\begin{aligned}
\frac{d r_{a}}{d t} & =\frac{1}{\tau_{r a}}\left(r_{a}^{s s}\left(u_{a}\left(r_{e}, r_{i}, I_{\mathrm{NMDA} a}\right)\right)-r_{a}\right) \\
\frac{d V_{a}}{d t} & =\frac{1}{\tau_{V a}}\left(V_{a}^{s s}\left(u_{a}\left(r_{e}, r_{i}, I_{\mathrm{NMDA} a}\right)\right)-V_{a}\right) \\
\frac{d I_{\mathrm{NMDA} a}}{d t} & =\frac{1}{\tau_{\mathrm{NMDA}}}\left(I_{\mathrm{NMDA} a}^{s s}\left(r_{e}, V_{a}\right)-I_{\mathrm{NMDA} a}\right),
\end{aligned}
$$

where $I_{\mathrm{NMDA} a}^{s s} \equiv I_{\mathrm{NMDA} a}^{s s, 0}\left(r_{e}, V_{a}\right)$ (renamed for notational consistency), and $r_{a}^{s s}\left(u_{a}\right), V_{a}^{s s}\left(u_{a}\right)$ are new functions that give the linearized dynamics of the firing rates and the membrane voltages. These functions are expressed as follows:

$$
\begin{gathered}
r_{a}^{s s}\left(u_{a}\right)=r_{a 0}+c_{r a}\left(u_{a}-u_{a 0}\right) \\
V_{a}^{s s}\left(u_{a}\right)=V_{a 0}+c_{V a}\left(u_{a}-u_{a 0}\right),
\end{gathered}
$$

where $c_{r a}$ and $c_{V a}$ are the derivatives of the instantaneous steady-state firing rate $r_{a}^{s s, 0}$ and the voltage $V_{a}^{s s, 0}$, respectively, taken by the input $u_{a}$ and evaluated at the equilibrium $S_{0}$ (where $c_{r a}$ is in units of $\mathrm{s}^{-1} \mathrm{~V}$ and $c_{V a}$ is unitless). For the rest of the paper, we will refer to (6) as the "unforced system."

The steady states of the system (6) can be found using selfconsistency conditions (see Appendix B). These conditions provide characteristic curves in the $\left(r_{e}, I_{\mathrm{NMDA} e}\right)$ plane, the intersections of which are the fixed points of (6):

$$
\begin{aligned}
r_{e} & =P_{r e}+Q_{r e}^{e} I_{\mathrm{NMDA} e}+Q_{r e}^{i} \bar{I}_{\mathrm{NMDA} i}\left(r_{e}, I_{\mathrm{NMDA} e}\right) \\
I_{\mathrm{NMDA} e} & =I_{\mathrm{NMDA} e}^{s s}\left(r_{e}, \bar{V}_{e}\left(r_{e}\right)\right),
\end{aligned}
$$

where $Q_{r a}^{b}, P_{r a}$ are constant coefficients depending on the synaptic weights; $\bar{V}_{a}\left(r_{a}\right), \bar{I}_{\mathrm{NMDA} i}\left(r_{e}, I_{\mathrm{NMDA} e}\right)$ are new functions that help to exclude all variables except for $r_{e}, I_{\mathrm{NMDA} e}$ from the self-consistency conditions (see Appendix B for more details).

We will refer to the curves defined by (8) as the $r_{e}$ curve and the $I_{\mathrm{NMDA}}$ curve, respectively. Configuration of these curves 
on the phase plane could provide important intuition about the system's behavior, which we discuss further in this paper.

\section{Forced oscillations of the fast linearized subsystem}

We now start exploring the system with external periodic forcing. Let us consider the system (6), forced by oscillatory inputs with the circular frequency $\omega$ and the complex-valued amplitudes of the inputs to the $E$ and $I$ populations equal to $h_{e}^{A}$ and $h_{i}^{A}$, respectively:

$$
\begin{aligned}
\frac{d r_{a}}{d t} & =\frac{1}{\tau_{r a}}\left(r_{a}^{s s}\left(u_{a}^{\mathrm{osc}}\left(r_{e}, r_{i}, I_{\mathrm{NMDA} a}, t\right)\right)-r_{a}\right) \\
\frac{d V_{a}}{d t} & =\frac{1}{\tau_{V a}}\left(V_{a}^{s s}\left(u_{a}^{\mathrm{osc}}\left(r_{e}, r_{i}, I_{\mathrm{NMDA} a}, t\right)\right)-V_{a}\right) \\
\frac{d I_{\mathrm{NMDA} a}}{d t}= & \frac{1}{\tau_{\mathrm{NMDA}}}\left(I_{\mathrm{NMDA} a}^{s s}\left(\operatorname{Re}\left(r_{e}\right), \operatorname{Re}\left(V_{a}\right)\right)\right. \\
& \left.-I_{\mathrm{NMDA} a}\right) \\
u_{a}^{\mathrm{osc}}\left(r_{e}, r_{i}, I_{\mathrm{NMDA} a}, t\right)= & J_{a e}^{\mathrm{AMPA}} r_{e}-J_{a i} r_{i} \\
& +I_{\mathrm{NMDA} a} / g_{m a}+h_{a}+h_{a}^{A} e^{i \omega t} .
\end{aligned}
$$

Using time scale separation, we can assume that the slow variables $I_{\mathrm{NMDA} e}$ and $I_{\mathrm{NMDA} i}$ do not get entrained by the external forcing and, for a given state $z_{S}$ of the slow subsystem, the dynamics $z_{F}(t)$ of the fast subsystem can be considered as sinusoidal oscillations around the corresponding equilibrium $z_{F}^{*}$ of the unforced fast subsystem.

We denote the complex-valued amplitudes of the firing rate and the voltage oscillations as $r_{a}^{A}$ and $V_{a}^{A}$, respectively. In our analyses, we will parametrize the system by $r_{a}^{A}$ (see the "Parameter selection" section below); however, we require explicit knowledge of $h_{a}^{A}$ for numerical simulations of the system (9). The value of $h_{a}^{A}$ that provides the entrained amplitudes $r_{a}^{A}$ could be expressed as follows:

$$
h_{a}^{A}=\frac{1}{c_{r a}} r_{a}^{A}\left(i \omega \tau_{r a}+1\right)-J_{a e}^{\mathrm{AMPA}} r_{e}^{A}+J_{a i} r_{i}^{A} .
$$

We will also need the relation between $V_{a}^{A}$ and $r_{a}^{A}$ for computing the forcing-induced shift of the time-averaged equilibrium. This relation is given by the following formula:

$$
V_{a}^{A}=r_{a}^{A} \frac{c_{V a}\left(1+i \omega \tau_{r a}\right)}{c_{r a}\left(1+i \omega \tau_{V a}\right)} .
$$

\section{Oscillation-induced shift of the slow subsystem (time-averaged) equilibrium}

We now analyze the influence of the forced oscillations of the fast subsystem on the dynamics of the slow subsystem, taking into account its nonlinearity.

First, let us introduce short notations for the functions that govern the dynamics of the NMDA currents [see (6) and (2)]:

$$
G_{a}\left(r_{e}, V_{a}, I_{\mathrm{NMDA} a}\right)=\frac{1}{\tau_{\mathrm{NMDA}}}\left(I_{\mathrm{NMDA} a}^{s s}\left(r_{e}, V_{a}\right)-I_{\mathrm{NMDA} a}\right) .
$$

By time scale separation, we can substitute $z_{F}$ by $z_{F}^{*}$, and thus express the unforced slow subsystem's dynamics as

$$
\begin{aligned}
\frac{d I_{\mathrm{NMDA} a}}{d t} & =G_{a}^{*}\left(I_{\mathrm{NMDA} e}, I_{\mathrm{NMDA} i}\right) \\
G_{a}^{*}\left(I_{\mathrm{NMDA} e}, I_{\mathrm{NMDA} i}\right) & =G_{a}\left(r_{e}^{*}\left(I_{\mathrm{NMDA} e}, I_{\mathrm{NMDA} i}\right), V_{a}^{*}\left(I_{\mathrm{NMDA} e}, I_{\mathrm{NMDA} i}\right), I_{\mathrm{NMDA} a}\right) .
\end{aligned}
$$

In the presence of the oscillatory input, we substitute $z_{F}$ by the sum of $z_{F}^{*}$ and the term that describes the entrained oscillations. The oscillation-perturbed slow dynamics are thus given by

$$
\begin{aligned}
\frac{d I_{\mathrm{NMDA} a}}{d t} & =G_{a}^{\mathrm{osc}}\left(I_{\mathrm{NMDA} e}, I_{\mathrm{NMDA} i}, t\right) \\
G_{a}^{\mathrm{osc}}\left(I_{\mathrm{NMDA} e}, I_{\mathrm{NMDA} i}, t\right) & =G_{a}\left(r_{e}^{*}+\left|r_{e}^{A}\right| \cos \left(\omega t+\psi_{r a}\right), V_{a}^{*}+\left|V_{a}^{A}\right| \cos \left(\omega t+\psi_{V a}\right), I_{\mathrm{NMDA} a}\right),
\end{aligned}
$$

where $\psi_{r a}=\arg r_{a}^{A}, \psi_{V a}=\arg V_{a}^{A}$.

We can now use averaging arguments [16] to estimate the effect of the forced oscillations on the slow dynamics. Let us introduce the NMDA currents $\tilde{I}_{\mathrm{NMDA}}$ and $\tilde{I}_{\mathrm{NMDA} i}$ averaged over one forcing period $T=2 \pi / \omega$. Their time-averaged dynamics are expressed as follows:

$$
\frac{d \tilde{I}_{\mathrm{NMDA} a}}{d \tilde{t}}=\frac{1}{T} \int_{\tilde{t}}^{\tilde{t}+T} G_{a}^{\mathrm{osc}}\left(I_{\mathrm{NMDA} e}, I_{\mathrm{NMDA} i}, t\right) d t .
$$

We can write down the full expression for $G_{a}^{\text {osc }}$ using (12), (2), and (3), expanding $g_{\text {NMDA }}$ (which enters in this expression) into Taylor series, up to $o\left(\left|r_{e}^{A}\right|^{2}\right)$. Then we can put the resulting estimate of $G_{a}^{\text {osc }}$ into (15) and take into account that all time-dependent oscillatory terms have zero integral over the period. This gives us the following autonomous system:

$$
\begin{aligned}
\frac{d \tilde{I}_{\mathrm{NMDA} a}}{d \tilde{t}} & =\tilde{G}_{a}\left(\tilde{I}_{\mathrm{NMDA} e}, \tilde{I}_{\mathrm{NMDA} i}\right) \\
\tilde{G}_{a}\left(\tilde{I}_{\mathrm{NMDA} e}, \tilde{I}_{\mathrm{NMDA} i}\right) & =G_{a}^{*}\left(\tilde{I}_{\mathrm{NMDA} e}, \tilde{I}_{\mathrm{NMDA} i}\right)+\frac{D_{a}\left(\tilde{r}_{e}, \tilde{V}_{a}\right)}{\tau_{\mathrm{NMDA}}} \\
D_{a}\left(\tilde{r}_{e}, \tilde{V}_{a}\right) & =\frac{1}{4} J_{a e}^{\mathrm{NMDA}}\left[2\left|r_{e}^{A}\right|\left|V_{a}^{A}\right| g_{\mathrm{NMDA}}^{\prime}\left(\tilde{V}_{a}\right) \cos \varphi_{a}+\tilde{r}_{e}\left|V_{a}^{A}\right|^{2} g^{\prime \prime}{ }_{\mathrm{NMDA}}\left(\tilde{V}_{a}\right)\right],
\end{aligned}
$$


where $\varphi_{a}=\psi_{V a}-\psi_{r e}$ is the phase lag between the voltage and the firing rate oscillations, $G_{a}^{*}$ is given above in (13), and $\tilde{r}_{a}, \tilde{V}_{a}$ are newly introduced time-averaged variables such that

$$
\begin{aligned}
\tilde{r}_{a} & \equiv r_{a}^{*}\left(\tilde{I}_{\mathrm{NMDA} e}, \tilde{I}_{\mathrm{NMDA} i}\right) \\
\tilde{V}_{a} & \equiv V_{a}^{*}\left(\tilde{I}_{\mathrm{NMDA} e}, \tilde{I}_{\mathrm{NMDA} i}\right) .
\end{aligned}
$$

The derivation of (16) is explained in more detail in Appendix B.

We can consider $\tilde{z}_{F} \equiv\left(\tilde{r}_{e}, \tilde{r}_{i}, \tilde{V}_{e}, \tilde{V}_{i}\right)$ as a slowly moving "center," around which the fast subsystem oscillates under the external forcing. From now on, we will refer to $\tilde{z}_{S} \equiv\left(\tilde{I}_{\mathrm{NMDA} e}, \tilde{I}_{\mathrm{NMDA} i}\right)$, together with $\tilde{z}_{F}$, as the time-averaged forced system. Dynamics of $\tilde{z}_{S}$ are given by (16), and $\tilde{z}_{F}$ functionally depends on $\tilde{z}_{s}$ via (17).

Let us now get a heuristic feel for the terms that influence the time-averaged forced system. The first term in the square brackets in (16) (containing $\left.\left|r_{e}^{A}\right|\left|V_{a}^{A}\right|\right)$ reflects the fact that the NMDA current depends both on the presynaptic firing rate and the postsynaptic voltage. The second term (containing $\left|V_{a}^{A}\right|^{2}$ ) reflects the fact that the NMDA current depends nonlinearly on the postsynaptic voltage. Our calculations have shown that, for the selected parameters, the first term dominates. Thus, the forcing-induced shift of the time-averaged equilibrium is mainly related to the joint effect of the presynaptic firing rate oscillations and the postsynaptic voltage oscillations, occurring with a small phase lag between them.

Now we can apply self-consistency analysis to the timeaveraged forced system and obtain the characteristic $\tilde{r}_{e}$ and $\tilde{I}_{\mathrm{NMDA} e}$ curves on the $\left(\tilde{r}_{e}, \tilde{I}_{\mathrm{NMDA} e}\right)$ plane [similar to what we did for the unforced system in (8)]:

$$
\begin{aligned}
\tilde{r}_{e}= & P_{r e}+Q_{r e}^{e} \tilde{I}_{\mathrm{NMDA} e}+Q_{r e}^{i} \bar{I}_{\mathrm{NMDA} i}\left(\tilde{r}_{e}, \tilde{I}_{\mathrm{NMDA} e}\right) \\
& +Q_{r e}^{i} D_{i}\left(\tilde{r}_{e}, \bar{V}_{i}\left(\bar{r}_{i}\left(\tilde{r}_{e}, \tilde{I}_{\mathrm{NMDA} e}\right)\right)\right) \\
\tilde{I}_{\mathrm{NMDA} e}= & I_{\mathrm{NMDA} e}^{s s}\left(\tilde{r}_{e}, \bar{V}_{e}\left(\tilde{r}_{e}\right)\right)+D_{e}\left(\tilde{r}_{e}, \bar{V}_{e}\left(\tilde{r}_{e}\right)\right)
\end{aligned}
$$

where $\bar{V}_{a}, \bar{r}_{i}, \bar{I}_{\mathrm{NMDA} i}$ are the functions that serve to express the self-consistency conditions in terms of $\tilde{r}_{e}, \tilde{I}_{\mathrm{NMDA}}$. These curves are parametrized by $r_{e}^{A}, r_{i}^{A}$ (via $D_{e}, D_{i}$ ), while $V_{e}^{A}, V_{i}^{A}$ could be factored out by using (11). Please see Appendix B for the details of the derivation.

Intersections of the $\tilde{r}_{e}$ curve and the $\tilde{I}_{\mathrm{NMDA} e}$ curve give the fixed points of the forced time-averaged system. One of these points that corresponds to the unforced equilibrium $S_{0}$ will be denoted as $\tilde{S}_{0} \equiv\left(\tilde{r}_{e 0}, \tilde{r}_{i 0}, \tilde{V}_{e 0}, \tilde{V}_{i 0}, \tilde{I}_{\mathrm{NMDA} e 0}, \tilde{I}_{\mathrm{NMDA} i 0}\right)$ further in the paper. The idea is to compare $\tilde{S}_{0}$ to its unforced counterpart $S_{0}$ [given by (8)] and thus to assess the oscillation-induced shift of the average firing rate $\Delta r_{e}=\tilde{r}_{e 0}-r_{e 0}$.

Let us estimate the shift $\Delta r_{e}$ under the assumption that the excitatory-to-excitatory (E-E) NMDA coupling is significantly stronger than the excitatory-to-inhibitory (E-I) NMDA coupling $\left(J_{i e}^{\mathrm{NMDA}} \ll J_{e e}^{\mathrm{NMDA}}\right)$. We were mainly interested in the excitatory effect of oscillations in this paper, so the EE NMDA connection plays the major role, while the E-I NMDA connection serves for stabilization purposes. First, recall that the first term dominates in the expression (16) for $D_{a}$. Second, note that $g_{\mathrm{NMDA}}(V)$ is close to linear for $V$ in the physiological range of voltages, so $g_{\mathrm{NMDA}}^{\prime}(V) \approx g_{\mathrm{NMDA}}^{\prime}\left(V_{a 0}\right)$. Third, as $J_{i e}^{\text {NMDA }}$ is small, we could assume that $D_{i} \approx 0$, $\bar{I}_{\mathrm{NMDA} i}\left(\tilde{r}_{e}, \tilde{I}_{\mathrm{NMDA} e}\right) \approx 0$. Given these simplifications, we could write the approximated version of (18):

$$
\begin{aligned}
\tilde{r}_{e} \approx & P_{r e}+Q_{r e}^{e} \tilde{I}_{\mathrm{NMDA} e} \\
\tilde{I}_{\mathrm{NMDA} e} \approx & I_{\mathrm{NMDA} e}^{s s}\left(\tilde{r}_{e}, \bar{V}_{e}\left(\tilde{r}_{e}\right)\right) \\
& +\frac{1}{2} J_{e e}^{\mathrm{NMDA}}\left|r_{e}^{A}\right|\left|V_{e}^{A}\right| g_{\mathrm{NMDA}}^{\prime}\left(V_{e 0}\right) \cos \varphi_{e} .
\end{aligned}
$$

Comparing (19) with the unforced equilibrium conditions (8), we conclude that

$$
\Delta r_{e} \approx \frac{1}{2} Q_{r e}^{e} J_{e e}^{\mathrm{NMDA}}\left|r_{e}^{A}\right|\left|V_{e}^{A}\right| g_{\mathrm{NMDA}}^{\prime}\left(V_{e 0}\right) \cos \varphi_{e}
$$

The expression (20) could be understood as follows. The external oscillatory input entrains the system, producing the excitatory firing rate and voltage oscillations with the amplitudes $\left|r_{e}^{A}\right|,\left|V_{e}^{A}\right|$ and the phase lag $\varphi_{e}$. These oscillations are fed into the slow nonlinear NMDA subsystem, shifting the steady-state NMDA current by $D_{e} \approx$ $\frac{1}{2} J_{e e}^{\mathrm{NMDA}}\left|r_{e}^{A}\right|\left|V_{e}^{A}\right| g_{\mathrm{NMDA}}^{\prime}\left(V_{e 0}\right) \cos \varphi_{e}$. This shift in the slow NMDA current is fed into the fast subsystem (which has the excitability given by $Q_{r e}^{e}$ ) and converted into the firing rate shift $\Delta r_{e} \approx Q_{r e}^{e} D_{e}$.

\section{E. Stability analysis of the unforced and the time-averaged periodically forced systems}

In the previous section, we provided expressions that could be used to estimate the oscillation-induced shift of the mean firing rate. However, we also need to identify the conditions under which such shift does not destabilize the system.

The unforced fast subsystem is stable when all the eigenvalues of the coupling matrix

$$
M_{r}=\left(\begin{array}{cc}
\frac{c_{r e} J_{e e}^{\mathrm{AMPA}}-1}{\tau_{r e}} & \frac{-c_{r e} J_{e i}}{\tau_{r e}} \\
\frac{c_{r i} J_{i e}^{\mathrm{APA}}}{\tau_{r i}} & \frac{-\left(c_{r i} J_{i j}+1\right)}{\tau_{r i}}
\end{array}\right)
$$

have negative real parts.

Stability of the slow unforced subsystem, in the case of $J_{i e}^{\mathrm{NMDA}} \neq 0$, could be determined by considering the Jacobian matrix of (13) calculated at the fixed point. The subsystem is stable if all the eigenvalues of this matrix have negative real parts. In the case of $J_{i e}^{\mathrm{NMDA}}=0$, the slow subsystem is stable, if $\left.\left(d G_{e}^{*}\left(I_{\mathrm{NMDAe}}\right) / d I_{\mathrm{NMDAe}}\right)\right|_{I_{\mathrm{NMDA} e \mathrm{O}}}<0$.

The asymptotic dynamics of the forced system could be considered as oscillations of the fast subsystem around the point $\tilde{z}_{F 0} \equiv\left(\tilde{r}_{e 0}, \tilde{r}_{i 0}, \tilde{V}_{e 0}, \tilde{V}_{i 0}\right)$ that functionally depends on the equilibrium $\tilde{z}_{S 0} \equiv\left(\tilde{I}_{\mathrm{NMDA} 0}, \tilde{I}_{\mathrm{NMDA} i 0}\right)$ of the time-averaged slow subsystem. Since the matrix $M_{r}$ is constant (does not depend on the slow variables), stability of the fast subsystem does not depend on the state of the slow subsystem. Hence, we are guaranteed that the forced oscillations will have a finite amplitude as long as the unforced system is stable (at least in the case when this amplitude is small enough). So we can focus exclusively on the stability of the time-averaged forced slow subsystem.

In the case of $J_{i e}^{\mathrm{NMDA}} \neq 0$, stability of the time-averaged forced slow subsystem is defined by the Jacobian matrix of (16) calculated at the fixed point. If all its eigenvalues have negative real parts, the subsystem is stable. In the case of $J_{i e}^{\mathrm{NMDA}}=0$, the slow subsystem is stable, if $d \tilde{G}_{e}\left(\tilde{I}_{\mathrm{NMDA} e}\right) /\left.d \tilde{I}_{\mathrm{NMDA} e}\right|_{\tilde{I}_{\mathrm{NMDA} e 0}}<0$. 
Of course, we note that the $\tilde{r}_{e}$ curve and the $\tilde{I}_{\mathrm{NMDA} e}$ curves should intersect; if they do not, the system would diverge towards unrealistically high firing rates, and the above linear stability conditions would not apply. However, if the unforced system has a steady state, the time-averaged forced system is also guaranteed to have one if the forcing amplitude is sufficiently small.

Let us transform the stability conditions for the case $J_{i e}^{\mathrm{NMDA}}=0$ to a form that is suitable for further geometrical analysis. To do so, we explicitly compute the derivatives $d G_{e}^{*} / d I_{\mathrm{NMDA} e}$ and $d \tilde{G}_{e} / d \tilde{I}_{\mathrm{NMDA} e}$, and take into account that $Q_{r e}^{e}>0$ for $J_{e e}^{\mathrm{AMPA}}$ being small enough. This gives us the following stability conditions for the unforced and for the time-averaged forced slow subsystems:

$$
\begin{array}{r}
\left.\frac{d I_{\mathrm{NMDA} e}^{s s}\left(r_{e}, \bar{V}_{e}\left(r_{e}\right)\right)}{d r_{e}}\right|_{r_{e 0}}<\frac{1}{Q_{r e}^{e}} \\
\left.\frac{d}{d \tilde{r}_{e}}\left(I_{\mathrm{NMDA} e}^{s s}\left(\tilde{r}_{e}, \bar{V}_{e}\left(\tilde{r}_{e}\right)\right)+D_{e}\left(\tilde{r}_{e}, \bar{V}_{e}\left(\tilde{r}_{e}\right)\right)\right)\right|_{\tilde{r}_{e 0}}<\frac{1}{Q_{r e}^{e}} .
\end{array}
$$

We further discuss (22) in Sec. III, where we consider the system with $J_{i e}^{\mathrm{NMDA}}=0$; we also provide geometrical interpretation for these conditions in terms of positions of the $r_{e}, \tilde{r}_{e}, I_{\mathrm{NMDA} e}, \tilde{I}_{\mathrm{NMDA} e}$ curves on the phase plane. In Sec. IV, where we consider the case of $J_{i e}^{\mathrm{NMDA}} \neq 0$, we check the stability conditions numerically.

\section{F. Parameter selection}

In this paper, we aimed at exploring the relationship between the forced oscillations and the induced shift of the time-averaged equilibrium. We were mainly interested in how this link depends on the synaptic weights. In fact, given the external tonic and oscillatory inputs, the synaptic weights also influence the steady state of the system, as well as the forced oscillations' amplitude. To disentangle these influences from the aforementioned relation of interest, we selected a priori reasonable values for the steady-state firing rates $\left(r_{e 0}, r_{i 0}\right)$, as well as for the firing rate amplitudes $\left(r_{e}^{A}, r_{i}^{A}\right)$. We then calculated the external input parameters (tonic and oscillatory) that provide the selected regime. For any synaptic weight combination we considered, this strategy allowed us to deal with the same slopes of the gain functions (dependent on the steady state) and the same forced amplitudes, both of which affect the oscillation-induced equilibrium shift.

After choosing the steady state, we determined the slopes of the gain functions $\left(c_{r a}, c_{V a}\right)$ by simulating individual leaky integrate-and-fire (LIF) neurons, one excitatory and one inhibitory (with standard parameters). Then we determined the time constants for the population firing rate and the mean membrane voltage dynamics ( $\tau_{r a}$ and $\tau_{V a}$, respectively) from simulated step responses of two (excitatory and inhibitory) uncoupled networks of LIF neurons. We note that we chose the LIF neural model as a paradigmatic minimal model of spiking; choosing another simple model such as the quadratic or the exponential integrate-and-fire model would not qualitatively change our results. More complex neural models account for nonlinear dynamics of various ion channels, which would additionally affect the oscillation-induced firing rate shift, while we aim to study the role of NMDA currents in producing this shift in isolation from these further mechanisms.

In this paper, we were mainly interested in the excitatory effect that the oscillations could exert on the system (positive rate shifts). In our model, this effect is mediated by the E-E NMDA connections. We surmised that strong E-I NMDA connections would lead to a net inhibitory effect of oscillations, while weak connections could potentially stabilize the system and help to achieve stronger excitation without loss of stability. To disentangle the effects of the E-E and the E-I NMDA connections, we considered two complementary cases. In the first case (model 1), NMDA inputs were located on the excitatory neurons only $\left(J_{i e}^{\mathrm{NMDA}}=0\right)$; in the second case (model 2), NMDA currents were located both on the excitatory and on the inhibitory neurons $\left(J_{i e}^{\mathrm{NMDA}} \neq 0\right)$. The synaptic weight $J_{i e}^{\mathrm{AMPA}}$ was decreased in model 2 (compared to model 1) in order to demonstrate that the fast subsystem could be made more excitable in the presence of the slow NMDA inhibition without loss of stability (which leads to a more pronounced forcing-induced shift of the mean excitatory firing rate). All the primary parameters of models 1 and 2, except $J_{i e}^{\mathrm{AMPA}}$ and $J_{i e}^{\mathrm{NMDA}}$, were set the same.

We have validated the selected parameters to make sure that they lead to physiologically reasonable properties of the system. We estimated strengths of external connections that could provide the tonic input with the selected characteristics (mean and variance). Then we checked that postsynaptic potentials corresponding both to the recurrent and to the estimated external connections are in the physiological range. We also confirmed that the unforced system operates in the subthreshold regime, and that the induced oscillations are relatively weak and do not cause crossing of the spike generation threshold. When these conditions are met, a low-dimensional model adequately captures the behavior of a corresponding spiking network.

The procedures for the parameter selection, derivation, and validation are described in more detail in Appendix A. The primary parameters are listed in Appendix A, Table I; the derived parameters, as well as characteristics of the equilibria, are listed in Table II.

\section{ANALYSIS OF THE SYSTEM WITH NMDA SYNAPSES ON THE EXCITATORY POPULATION ONLY (MODEL 1)}

Let us first analyze the oscillation-induced mean firing rate changes in a network where the NMDA inputs are located on the excitatory neurons only $\left(J_{i e}^{\mathrm{NMDA}}=0\right)$. We take a geometric approach and consider the phase plane where we plot the $r_{e}$ and $I_{\mathrm{NMDA} e}$ curves to determine the existence and stability of the equilibrium in the time-averaged forced system. Next, we confirm the predictions of the phase plane analysis by a numerical simulation of the system.

Given the condition $J_{i e}^{\mathrm{NMDA}}=0$, Eqs. (8) for the $r_{e}$ and $I_{\mathrm{NMDA} e}$ curves of the unforced system are expressed as follows:

$$
\begin{aligned}
r_{e} & =P_{r e}+Q_{r e}^{e} I_{\mathrm{NMDA} e} \\
I_{\mathrm{NMDA} e} & =I_{\mathrm{NMDA} e}^{s s}\left(r_{e}, \bar{V}_{e}\left(r_{e}\right)\right),
\end{aligned}
$$


and the similar Eqs. (18) for the $\tilde{r}_{e}$ and $\tilde{I}_{\mathrm{NMDAe}}$ curves of the time-averaged forced system could be expressed as follows:

$$
\begin{aligned}
\tilde{r}_{e} & =P_{r e}+Q_{r e}^{e} \tilde{I}_{\mathrm{NMDA} e} \\
\tilde{I}_{\mathrm{NMDA} e} & =I_{\mathrm{NMDA} e}^{s s}\left(\tilde{r}_{e}, \bar{V}_{e}\left(\tilde{r}_{e}\right)\right)+D_{e}\left(\tilde{r}_{e}, \bar{V}_{e}\left(\tilde{r}_{e}\right)\right) .
\end{aligned}
$$

From (23) and (24), it is seen that the $r_{e}$ and $\tilde{r}_{e}$ curves are identical and both are represented by a straight line (we refer to it as the $r_{e} / \tilde{r}_{e}$ curve).

Let us now examine the phase plane shown in Fig. 1(a): the solid black line represents the $r_{e} / \tilde{r}_{e}$ curve, the solid blue line represents the $I_{\mathrm{NMDAe}}$ curve, and the solid red line represents the $\tilde{I}_{\text {NMDAe }}$ curve. The point $S_{0}$ is the equilibrium $\left(r_{e 0}, I_{\mathrm{NMDA} e 0}\right)$ of the unforced system, and $\tilde{S}_{0}$ is the equilibrium $\left(\tilde{r}_{e 0}, \tilde{I}_{\mathrm{NMDA} e 0}\right)$ of the time-averaged forced system. The unstable fixed point of the time-averaged forced system is denoted by $\tilde{S}_{1}$. A zoomed region of the phase plane containing the equilibrium points is presented in Fig. 1(b) [this region is denoted by the gray rectangle in Fig. 1(a)]. The dashed red line is the line tangent to the $\tilde{I}_{\mathrm{NMDA}}$ curve that passes through $S_{0}$ (the tangency point at the $\tilde{I}_{\mathrm{NMDAe}}$ curve is denoted by $A$ ). The importance of this line is discussed below.

Taking into account the stability conditions (22), we can see a simple geometrical interpretation for stability of the slow subsystem of the unforced and of the time-averaged forced system. The right-hand parts of the inequalities (22) give the slope of the $r_{e} / \tilde{r}_{e}$ curve. The left-hand parts define the slopes of the lines tangent to the $I_{\mathrm{NMDAe}}$ curve at $S_{0}$ (first inequality) and to the $\tilde{I}_{\mathrm{NMDA}}$ curve at $\tilde{S}_{0}$ (second inequality). Consequently, the slow unforced subsystem is stable at $S_{0}$, if the $r_{e} / \tilde{r}_{e}$ curve is steeper than the $I_{\mathrm{NMDAe}}$ curve at $S_{0}$. Similarly, the slow time-averaged forced subsystem is stable at $\tilde{S}_{0}$, if the $r_{e} / \tilde{r}_{e}$ curve is steeper than the $\tilde{I}_{\mathrm{NMDAe}}$ curve at $\tilde{S}_{0}$. From Fig. 1 , one can see that both of these conditions are satisfied, so both $S_{0}$ and $\tilde{S}_{0}$ are the stable fixed points of the corresponding subsystems. We can also apply the arguments above to the fixed point $\tilde{S}_{1}$ and conclude that it is unstable, since the $\tilde{I}_{\mathrm{NMDA}}$ curve is steeper than the $r_{e} / \tilde{r}_{e}$ curve at this point.

There is an important consequence of the stability conditions. As mentioned above, if $S_{0}$ is stable, then the $r_{e} / \tilde{r}_{e}$ curve (which is a straight line) is steeper than the $I_{\mathrm{NMDA}}$ curve. Taking into account that the $\tilde{I}_{\mathrm{NMDA}}$ curve is above the $I_{\mathrm{NMDA}}$ curve, it follows that $\tilde{r}_{e 0}>r_{e 0}$, i.e., the oscillatory forcing has an excitatory effect on the system.

We can also formulate the geometric conditions for the existence of the time-averaged forced equilibrium $\tilde{S}_{0}$. The dynamics of the forced system is bounded only if the $r_{e} / \tilde{r}_{e}$ curve intersects with the $\tilde{I}_{\mathrm{NMDA}}$ curve, which implies that the $r_{e} / \tilde{r}_{e}$ curve should rise steeper than the line passing through $S_{0}$ and tangent to the $\tilde{I}_{\mathrm{NMDAe}}$ curve [the latter is represented by the dashed red line $\left(S_{0} A\right)$ in Fig. 1(b)]. As we make the fast subsystem more excitable (by increasing $J_{e e}^{\mathrm{AMPA}}, J_{i i}$ or decreasing $\left.J_{i e}^{\mathrm{AMPA}}, J_{e i}\right)$, the angle of the $r_{e} / \tilde{r}_{e}$ curve decreases, and eventually this curve coincides with the $\left(S_{0} A\right)$ line, which corresponds to the saddle-node bifurcation in the time-averaged forced system (the stable fixed point $\tilde{S}_{0}$ merges with the unstable fixed point $\tilde{S}_{1}$ at the point $A$ ). With
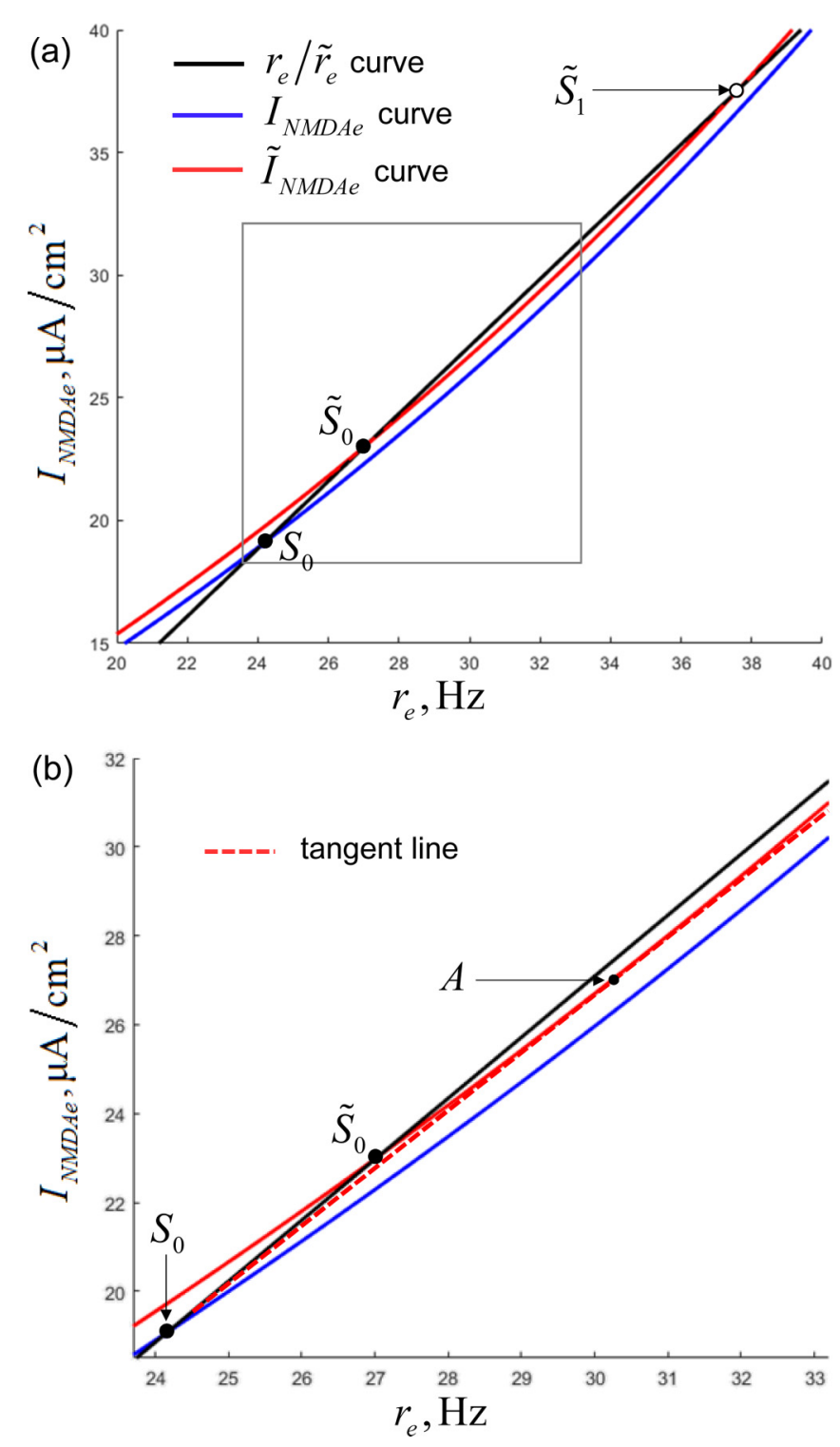

FIG. 1. A geometrical approach to finding steady states of the unforced and of the time-averaged forced systems, applied to model 1 (containing NMDA synapses on the excitatory population only). The black line $\left(r_{e} / \tilde{r}_{e}\right.$ curve $)$ and the blue line $\left(I_{\mathrm{NMDA} e}\right.$ curve $)$ are defined by two self-consistency conditions for the unforced system that link the excitatory firing rate $r_{e}$ with the NMDA current $I_{\mathrm{NMDA} e}$ received by the excitatory population. The intersection of these curves gives the steady state of the unforced system $S_{0}$. Analogous self-consistency conditions for the time-averaged forced system are given by the same black line $\left(r_{e} / \tilde{r}_{e}\right.$ curve $)$ and by the red line $\left(\tilde{I}_{\mathrm{NMDA} e}\right.$ curve $)$; they link the averaged firing rate $\tilde{r}_{e}$ with the averaged NMDA current $\tilde{I}_{\text {NMDAe }}$. The intersections of these curves provide the stable fixed point $\tilde{S}_{0}$ and the unstable fixed point $\tilde{S}_{1}$ of the time-averaged forced system. Part of the phase plane marked by the rectangle in panel (a) is zoomed in in panel (b). The red dashed line in (b) is the tangent line to the $\tilde{I}_{\mathrm{NMDAe}}$ curve containing $S_{0} ; A$ is the corresponding tangency point. Since the $r_{e} / \tilde{r}_{e}$ curve is a straight line, the rightmost possible position of $\tilde{S}_{0}$ (under variations of the fast synaptic weights) is the point $A$, which strongly limits the possible forcing-induced mean excitatory firing rate shift. 

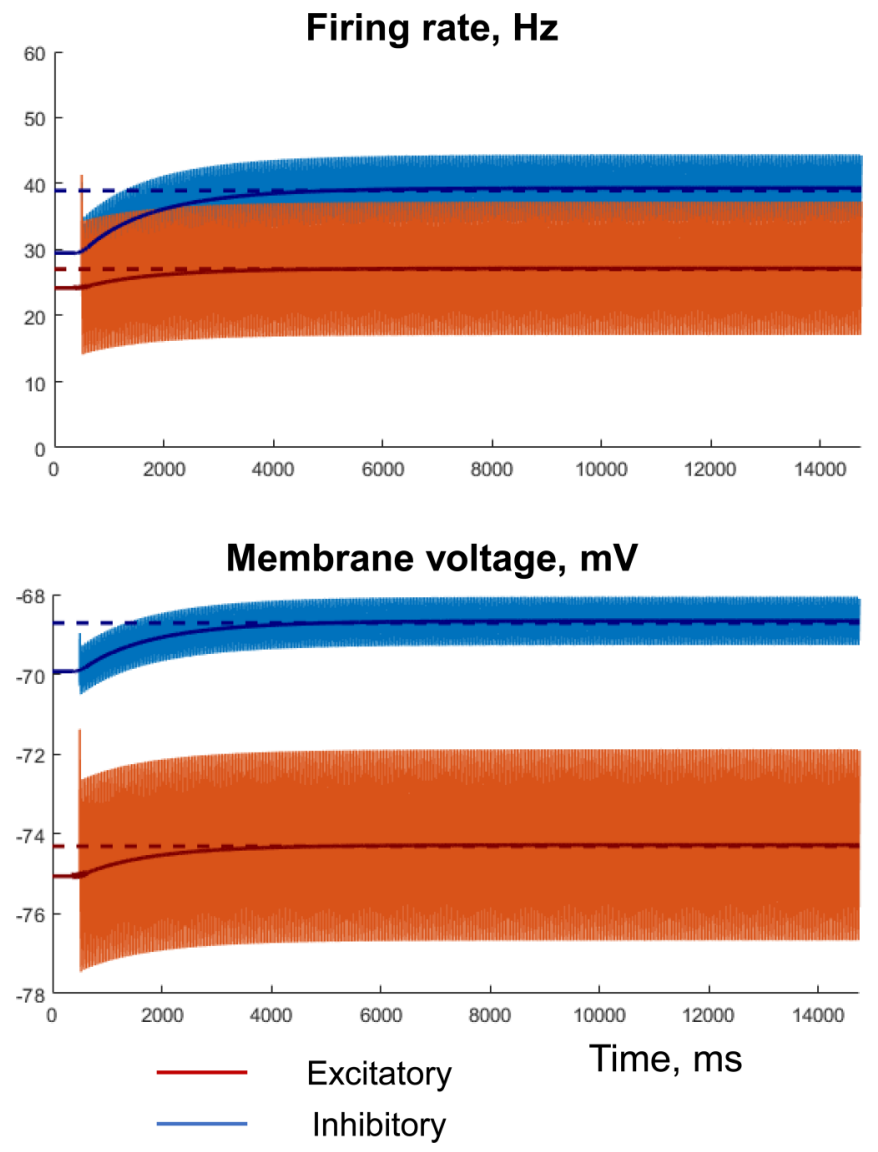

FIG. 2. Results of a simulation of model 1 (containing NMDA synapses on the excitatory population only). Red, excitatory population; blue, inhibitory population. Thin solid lines, simulated traces; thick solid lines, simulated traces averaged over $250 \mathrm{~ms}$; thick dashed lines, predictions of the graphical analysis. The simulation time step was $\Delta t=0.1 \mathrm{~ms}$. The oscillation-induced excitatory firing rate shift is small [see the difference between the initial point of the red trace in the top panel (unforced firing rate) and the dashed red line (time-averaged firing rate under the forcing)].

the further increase of excitability, the fixed points $\tilde{S}_{0}$ and $\tilde{S}_{1}$ disappear, and the dynamics of the forced system diverges.

To confirm the predictions of the geometrical analysis, we performed a numerical simulation of the low-dimensional system described by (9). The simulation started from the unforced steady state; the periodic external forcing was turned on at $t_{\mathrm{osc}}=200 \mathrm{~ms}$. The simulation was performed during $T=15$ s. As we can see in Fig. 2, the averaged simulated firing rates and population voltages (thick solid lines in the upper and lower panels, respectively) approach the values that are close to the ones predicted from the aforementioned phase plane analysis (dashed horizontal lines). In agreement with Fig. 1, the mean excitatory firing rate shift is small (about $3 \mathrm{~Hz}$ ).

\section{ANALYSIS OF THE SYSTEM WITH NMDA SYNAPSES ON THE EXCITATORY AND INHIBITORY POPULATIONS (MODEL 2)}

In this section, we analyze model 2 that contains NMDA synapses both on the excitatory and on the inhibitory neurons.

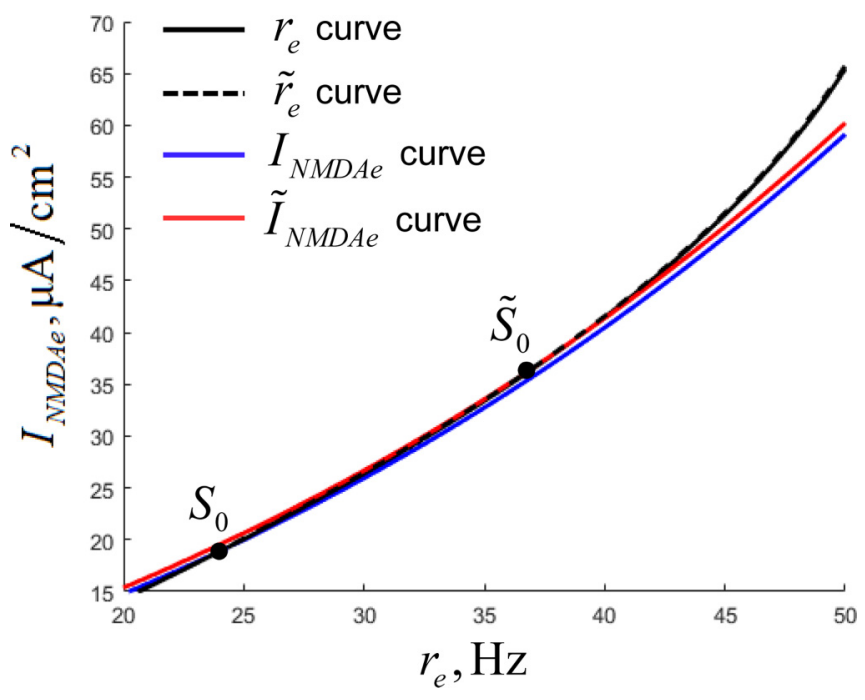

FIG. 3. A geometrical approach to finding steady states of the unforced and of the time-averaged forced systems, applied to model 2 (containing NMDA synapses both on the excitatory and the inhibitory populations). The solid black line ( $r_{e}$ curve) and the blue line $\left(I_{\mathrm{NMDA}}\right.$ curve) are defined by two self-consistency conditions for the unforced system that link the excitatory firing rate $r_{e}$ with the NMDA current $I_{\mathrm{NMDA}}$ received by the excitatory population; intersection of these curves gives the steady state of the unforced system $S_{0}$. The dashed black line ( $\tilde{r}_{e}$ curve) and the red line $\left(\tilde{I}_{\mathrm{NMDA} e}\right.$ curve) are defined by analogous self-consistency conditions for the time-averaged forced system (linking the averaged firing rate $\tilde{r}_{e}$ with the averaged NMDA current $\tilde{I}_{\mathrm{NMDA} e}$ ); intersection of these curves gives the steady state of the time-averaged forced system $\tilde{S}_{0}$. Note that the nonlinearity of the $\tilde{r}_{e}$ curve yields a large distance between $S_{0}$ and $\tilde{S}_{0}$ and, thus, a large oscillation-induced mean excitatory firing rate shift.

First, we note that the E-I NMDA coupling $J_{i e}^{\mathrm{NMDA}}$ is much weaker in the model than the E-E NMDA coupling $J_{e e}^{\mathrm{NMDA}}$, so the main effect of the external forcing on the system is still excitatory. The role of the E-I NMDA coupling here is to make the system more robust, which allows the fast subsystem to be more excitable without having divergent dynamics in the presence of the external forcing. In model 2 , the fast subsystem was made more excitable compared to model 1 by decreasing the E-I AMPA coupling $J_{i e}^{\mathrm{AMPA}}$.

The phase plane for model 2 is presented in Fig. 3. The legend is the same as in Fig. 1(a) (which represents the same picture for model 1), with the difference that the $\tilde{r}_{e}$ curve now differs from the $r_{e}$ curve; the $\tilde{r}_{e}$ curve is represented in Fig. 3 by the dashed black line, and the $r_{e}$ curve is represented by the solid black line. Comparing Figs. 1 and 3, one can see two major differences between model 1 and model 2. First, the $\tilde{r}_{e}$ curve for model 2 is concave (as opposed to being linear for model 1). Consequently, the position of the time-averaged forced equilibrium $\tilde{S}_{0}$ in model 2 is not limited as it is for model 1 by the point, denoted in Fig. 1(b) as $A$ [which is the point on the $\tilde{I}_{\text {NMDAe }}$ curve such that the line $\left(S_{0} A\right)$ is tangent to the $\tilde{I}_{\mathrm{NMDA}}$ curve].

The second difference is that in model 2 the $\tilde{r}_{e}$ curve separates rapidly from the $\tilde{I}_{\mathrm{NMDA}}$ curve in the region above $\tilde{S}_{0}$, so there is no unstable point $\tilde{S}_{1}$ near $\tilde{S}_{0}$. Thus, we 

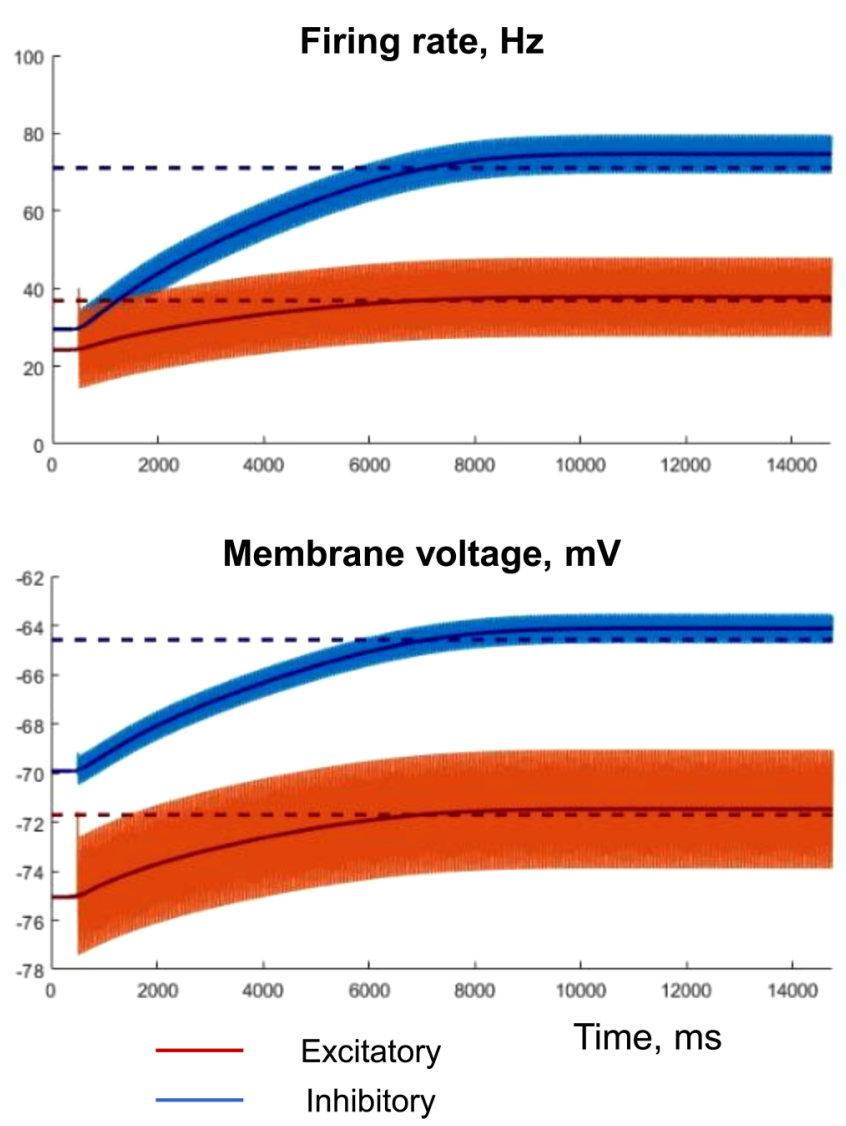

FIG. 4. Results of a simulation of model 2 (containing NMDA synapses both on the excitatory and the inhibitory populations). Red, excitatory population; blue, inhibitory population. Thin solid lines, simulated traces; thick solid lines, simulated traces averaged over $250 \mathrm{~ms}$; thick dashed lines, predictions of the graphical analysis. The simulation time step was $\Delta t=0.1 \mathrm{~ms}$. The oscillation-induced excitatory firing rate shift is relatively large [see the difference between the initial point of the red trace in the top panel (unforced firing rate) and the dashed red line (time-averaged firing rate under the forcing)].

expect that no saddle-node bifurcation (and, consequently, no divergent dynamics) will occur if we make the fast subsystem more excitable (as we observed in model 1). In the following section, we demonstrate that it is indeed the case, and that the system loses its stability via a supercritical Hopf bifurcation, and starts to generate very slow oscillations.

We should note that stability of $S_{0}$ and $\tilde{S}_{0}$ for the case of $J_{i e}^{\mathrm{NMDA}} \neq 0$ does not follow from simple geometrical considerations, so it should be checked explicitly by numerically calculating the eigenvalues of the Jacobian matrices of (13) and (16), respectively (evaluated at the corresponding fixed points). We did it for model 2 and confirmed that both $S_{0}$ and $\tilde{S}_{0}$ are stable.

We confirmed the predictions of the phase plane analysis for model 2 by numerical simulation of the system (9), using the same simulation parameters as for model 1 (starting from the unforced equilibrium, turning on the periodic forcing, 15-s simulation). The results of the simulation are represented in Fig. 4; the legend is the same as in Fig. 2. One can see that the results are in a good agreement with the predictions, and that the excitatory mean firing rate shift is considerably large (about $13 \mathrm{~Hz}$ ).

The mean firing rate shift in Fig. 4 unfolds during a relatively long time period after the beginning of the oscillatory input. Arguably, this may limit the possible physiological role of the NMDA-based mechanism we lay out above in fast cognitive operations such as perceptual decision making. To check whether it is possible to overcome this limitation, we performed simulations of model 2 with decreased NMDA time constant $\tau_{\mathrm{NMDA}}$. The results for $\tau_{\mathrm{NMDA}}=50$ and $100 \mathrm{~ms}$ are presented in Figs. 5(a) and 5(b), respectively; the result for the original value $\tau_{\mathrm{NMDA}}=200 \mathrm{~ms}$ is presented in Fig. 5(c) for comparison [identical to the upper panel of Fig. 4]. We can see that the time-averaged excitatory firing rate rises much steeper for smaller $\tau_{\mathrm{NMDA}}$ values after turning on the oscillatory input. For example, a $10-\mathrm{Hz}$ increase occurs in $610 \mathrm{~ms}$ for $\tau_{\mathrm{NMDA}}=$ $50 \mathrm{~ms}$, compared to $3910 \mathrm{~ms}$ for $\tau_{\mathrm{NMDA}}=200 \mathrm{~ms}$; a $5-\mathrm{Hz}$ increase occurs in $265 \mathrm{~ms}$ for $\tau_{\mathrm{NMDA}}=50 \mathrm{~ms}$, compared to $1475 \mathrm{~ms}$ for $\tau_{\mathrm{NMDA}}=200 \mathrm{~ms}$. Given that $\tau_{\mathrm{NMDA}}=50 \mathrm{~ms}$ is still a reasonable value for the NMDA time constant, the obtained results broaden the physiological applicability of the proposed NMDA-based mechanism.

For the original model 2, there is a slight deviation between the analytical prediction of the oscillation-induced firing rate shift and the simulation results [see Figs. 4 and $5(\mathrm{c})]$. This is caused by the fact that the ratio between the slow and the fast time scales is large, but not infinite (as the slow-fast approximation suggests). Thus, the oscillatory input entrains weak oscillations of the NMDA currents, which are not accounted for in the analytical prediction. To ground this explanation, we performed a simulation of model 2 with largely increased NMDA time constant (which is closer to an ideal slow-fast system); the NMDA-related synaptic weights were appropriately rescaled. The result is presented in Fig. 5(d), and it is seen that the prediction in this case is almost perfect. In contrast, the simulation results for smaller NMDA time constants show stronger deviation towards higher values from the corresponding predictions [Figs. 5(a) and 5(b)] since the oscillatory entrainment of the NMDA currents is more pronounced when these currents are faster.

\section{DEPENDENCE OF THE STEADY-STATE SHIFT ON PARAMETERS}

The major goal of our paper is to explore to what extent the nonlinear NMDA coupling could, in principle, account for oscillatory-induced shifts of the mean level of activity in a neural population. In the previous sections, we derived analytic expressions describing this shift and explored the system behavior for two specific combinations of parameters (model 1 and model 2). This provided us some intuitions about links between the system parameters and the firing rate shift. We also observed that the shift is limited by stability issues (if it is too strong, the shifted equilibrium could disappear or become unstable). In this section, we explore these relations more systematically.

We tracked how the mean firing rate shift $\Delta r_{e 0}=\tilde{r}_{e 0}-r_{e 0}$ depends on the slow NMDA synaptic weights $J_{e e}^{\mathrm{NMDA}}, J_{i e}^{\mathrm{NMDA}}$ and on the amplitudes of the forced firing rate oscillations 

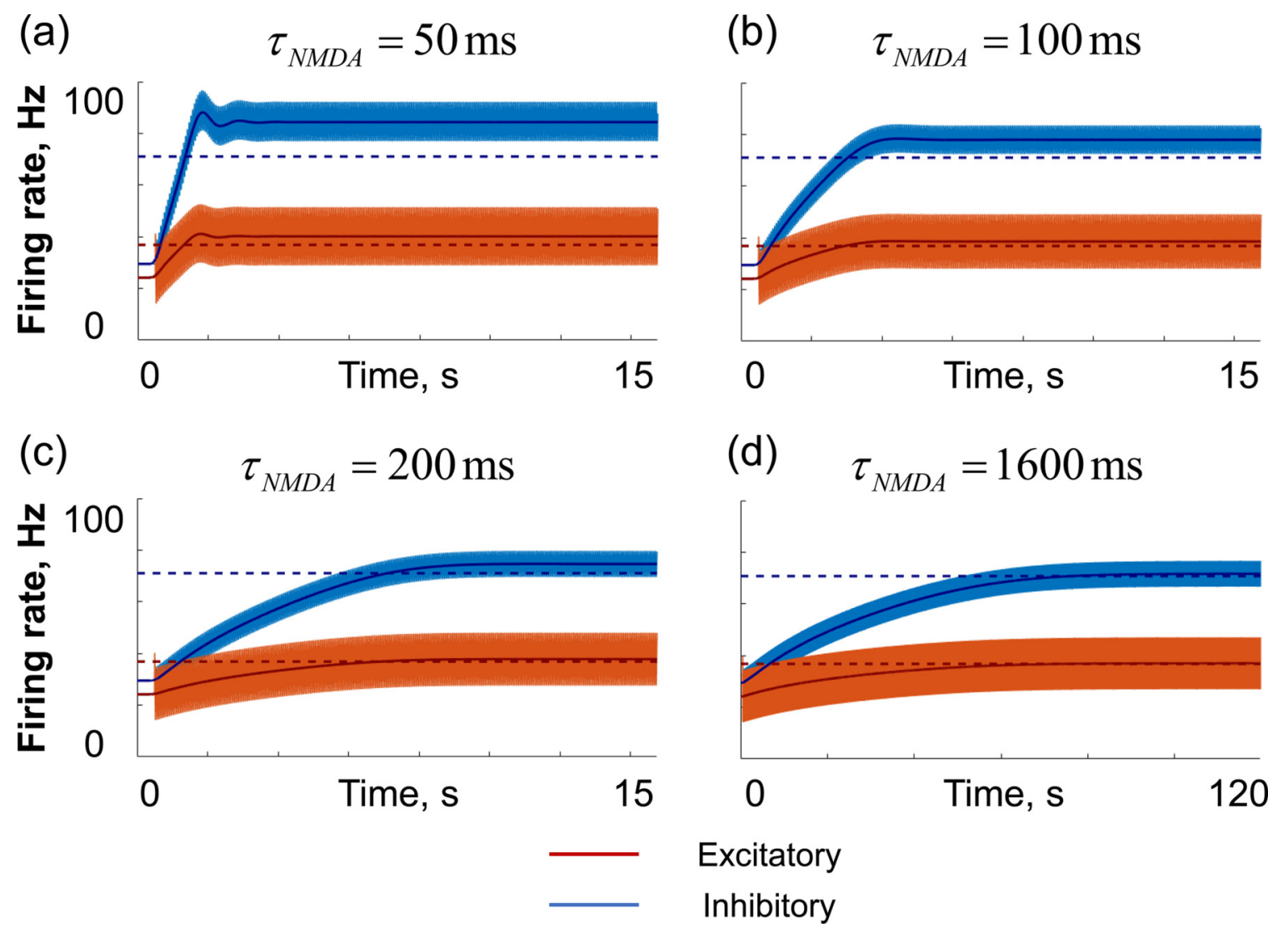

FIG. 5. Results of simulations of model 2 (containing NMDA synapses both on the excitatory and the inhibitory populations) with various NMDA time constants. Red, excitatory population; blue, inhibitory population. Thin solid lines, simulated traces; thick solid lines, simulated traces averaged over $250 \mathrm{~ms}$; thick dashed lines, predictions of the graphical analysis. The simulation time step was $\Delta t=0.1 \mathrm{~ms}$. (a), (b) NMDA time constant is decreased compared to the original value. (c) NMDA time constant equals to the original value. (d) NMDA time constant is largely increased compared to the original value. NMDA-related synaptic weights were rescaled to achieve the same NMDA currents in all four cases. One could see that for smaller NMDA time constants the oscillation-induced increase of the mean firing rate is steeper [note a different time scale in (d)], and its steady-state value deviates more strongly from the analytical predictions towards higher values.

$\left|r_{e}^{A}\right|,\left|r_{i}^{A}\right|$, given zero phase lag between these oscillations (see Fig. 6). We also examined how this shift depends on the fast excitatory synaptic weights $J_{e e}^{\mathrm{AMPA}}, J_{i e}^{\mathrm{AMPA}}$ and on the fast inhibitory synaptic weights $J_{e i}, J_{i i}$ (see Fig. 7). As we mentioned above, the shift is, in principle, delimited by the system instabilities (see the black, green, and red lines in Figs. 6 and 7).

As we can see from the figures, $\Delta r_{e 0}$ increases with $J_{e e}^{\mathrm{NMDA}}, J_{e e}^{\mathrm{AMPA}}, J_{i i}$ (i.e., when there is more excitation in the system; note that $J_{i i}$ determines inhibition of inhibition, so, effectively, it controls excitation) and decreases with $J_{i e}^{\mathrm{NMDA}}, J_{i e}^{\mathrm{AMPA}}, J_{e i}$ (i.e., when there is more inhibition in the system). Also, the rate shift increases with $\left|r_{e}^{A}\right|$ (when the excitatory population is more strongly entrained by the external forcing) and decreases with $\left|r_{i}^{A}\right|$ (when the inhibitory population is more strongly entrained). As we can also see, the highest firing rate shift $\Delta r_{e 0}$ is always observed near the borders of instability of the slow subsystem. Interestingly, there is an optimal combination of $J_{e e}^{\mathrm{NMDA}}, J_{i e}^{\mathrm{NMDA}}$, giving the maximal rate shift [see Fig. 6(a)].

In the previous sections, we demonstrated that the firing rate shift $\Delta r_{e 0}$ grows with increasing the excitability of the fast subsystem, i.e., its ability to convert the slow NMDA current shift to the firing rate shift [e.g., see the approximate result
(20)]. Geometrically, higher excitability results in a lower slope of the $\tilde{r}_{e}$ curve, so it intersects with the $\tilde{I}_{\text {NMDAe }}$ curve at a higher firing rate. However, excessive excitability may lead to a disappearance of this intersection and to divergence of the forced dynamics. We also provided geometrical arguments that having nonzero E-I NMDA coupling $J_{i e}^{\mathrm{NMDA}}$ allows one to increase the fast subsystem excitability (and, thus, obtain a higher shift) without destabilizing the system. We confirmed this by analyzing two distinct parameter combinations - one with $J_{i e}^{\mathrm{NMDA}}=0$ (model 1$)$, and another with $J_{i e}^{\text {NMDA }} \neq 0$ (model 2$)$.

Here we explore in more detail how the E-I NMDA coupling strength and the fast subsystem excitability jointly define the firing rate shift. The goal is to explore their interaction in detail to understand under which conditions the highest possible shift could be achieved. The excitability of the fast subsystem could be controlled by changing any of the fast weights; here we chose $J_{i e}^{\text {AMPA }}$ for this purpose. Thus, we described the dependence of the system's behavior on the slow E-I NMDA coupling weight $J_{i e}^{\text {NMDA }}$ and on the fast E-I AMPA coupling weight $J_{i e}^{\mathrm{AMPA}}$. We analyzed the bifurcations that occurred in the time-averaged forced system when $J_{i e}^{\mathrm{NMDA}}$ and $J_{i e}^{\mathrm{AMPA}}$ were varied and explored the consequences of these 

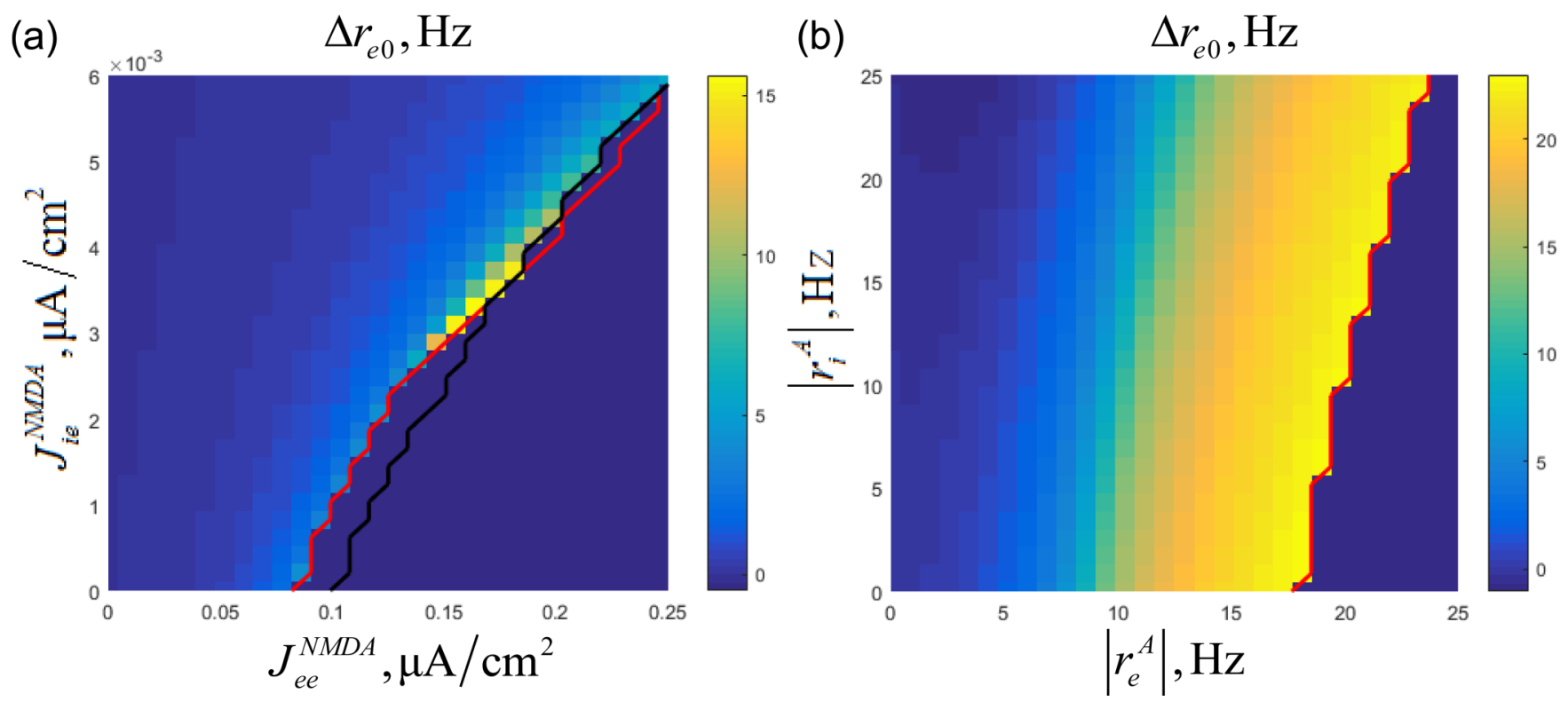

FIG. 6. Dependence of the forcing-induced shift of the time-averaged equilibrium $\Delta r_{e 0}$ on the following parameters: (a) the slow synaptic weights and (b) the forced amplitudes. Black line, the border of stability of the unforced slow subsystem; red line, the border of stability of the time-averaged forced system. The instability region is filled by the dark-blue color. Note the optimal parameter combination in panel (a) that yields the maximal $\Delta r_{e 0}$.

bifurcations for the ability of periodic forcing to excite the system without destabilizing it.

Let us start by briefly describing the main features of the bifurcation structure of the time-averaged forced system. In Fig. 8(a), the two-dimensional (2D) bifurcation diagram is presented, with $J_{i e}^{\mathrm{AMPA}}$ on the horizontal axis and $J_{i e}^{\mathrm{NMDA}}$ on the vertical axis. In Fig. 8(b), a region of the diagram is zoomed in. The black line corresponds to saddle-node bifurcations: two fixed points merge and disappear as the line $\mathrm{AB}$ or $\mathrm{BC}$ is crossed from the right to the left, and as the line $\mathrm{BC}$ is crossed from the left to the right. In the region above the green line, one of the fixed points is a focus, so the system demonstrates either damped or sustained slow oscillations. This focus is stable to the right of the red line CG and unstable to the left of it; the red line CG itself corresponds to the supercritical Hopf bifurcation. The intersection of the $\mathrm{AB}$ and $\mathrm{CG}$ lines is denoted by $\mathrm{E}$ (its importance will become clear later in this section). (a)

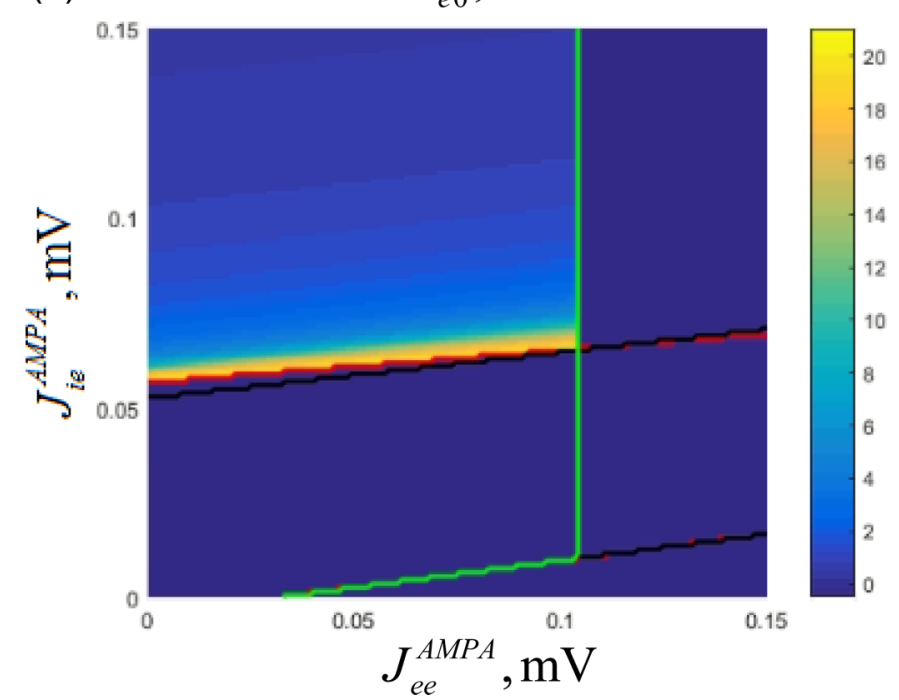

(b)

$\Delta r_{e 0}, \mathrm{~Hz}$

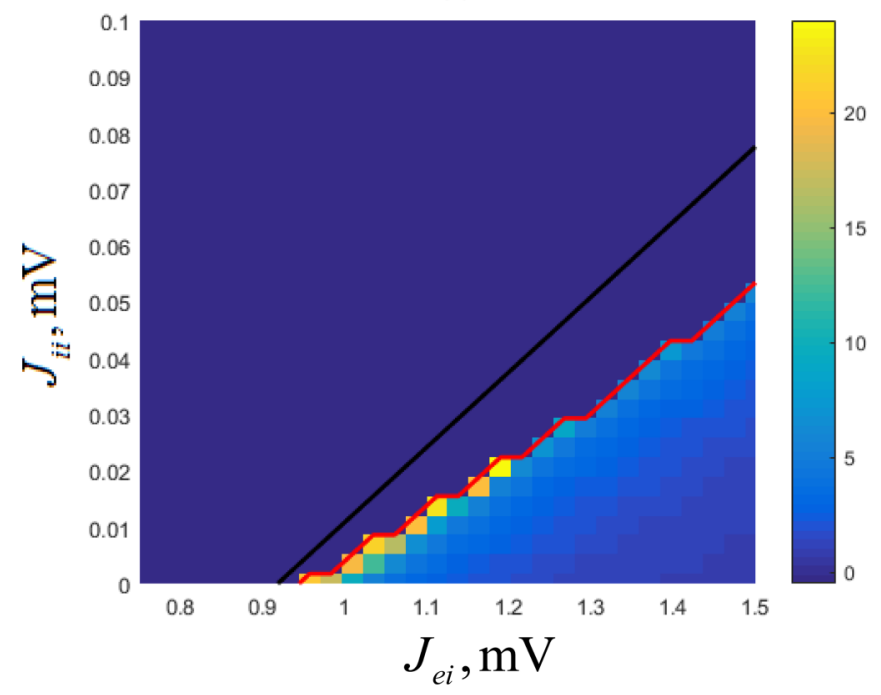

FIG. 7. Dependence of the forcing-induced shift of the time-averaged equilibrium $\Delta r_{e 0}$ on the following parameters: (a) the fast excitatory synaptic weights and (b) the fast inhibitory synaptic weights. Black line, the border of stability of the unforced slow subsystem; red line, the border of stability of the time-averaged forced system; green line, the border of stability of the fast subsystem. The instability region is filled by the dark-blue color. 
(a)
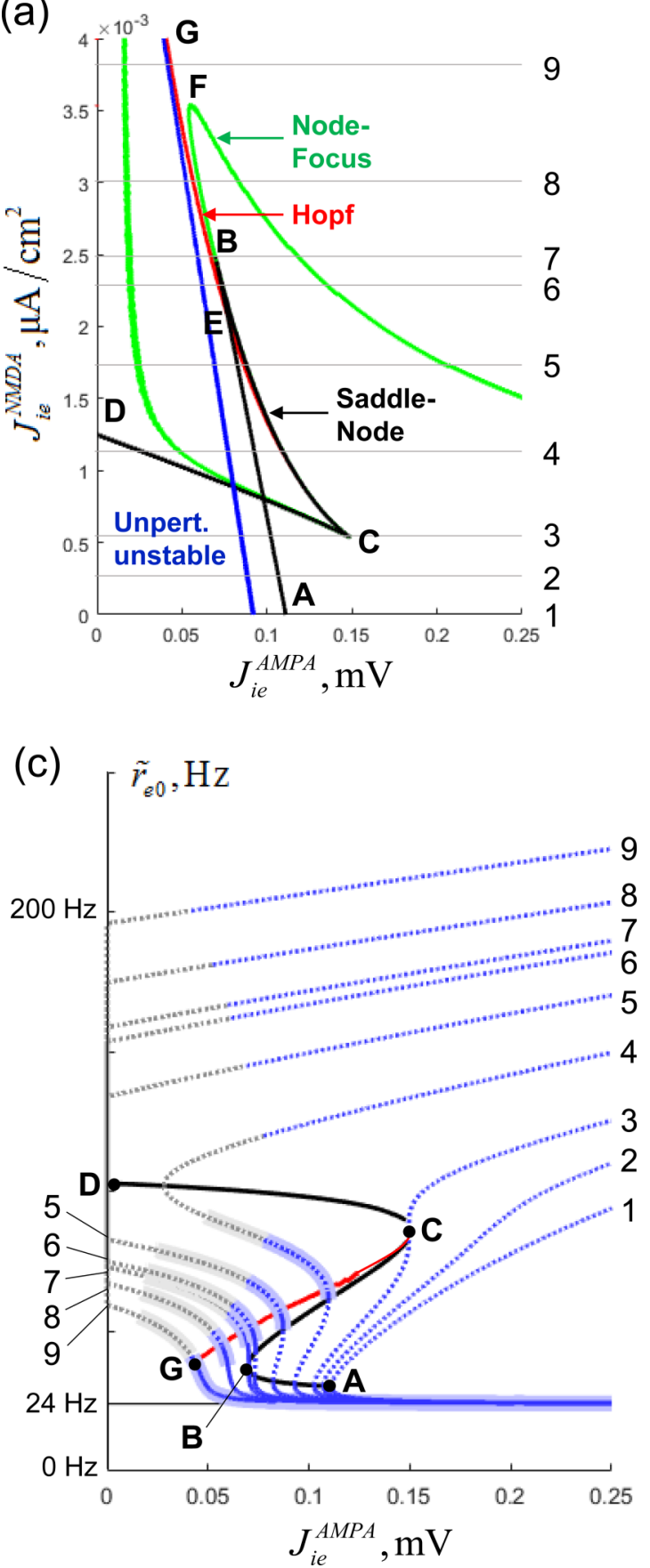

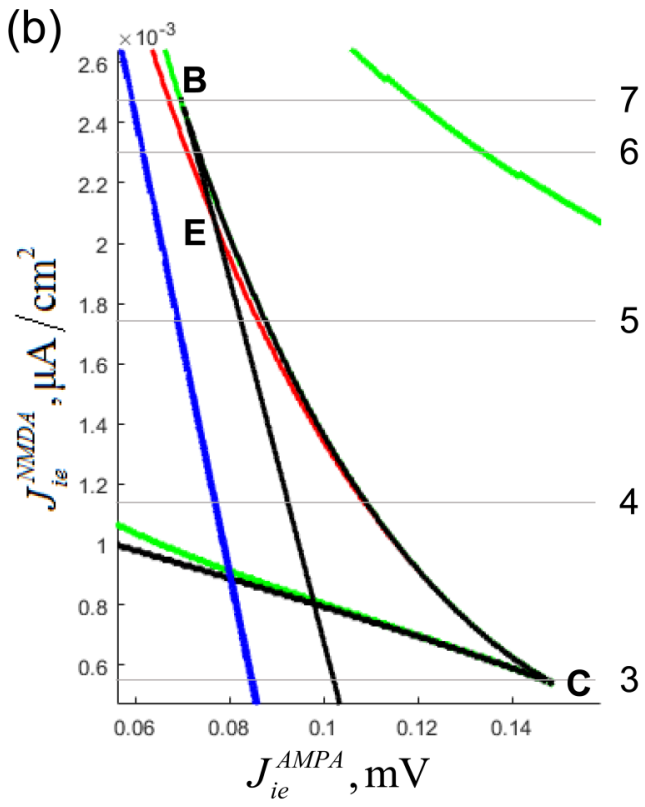

(d)

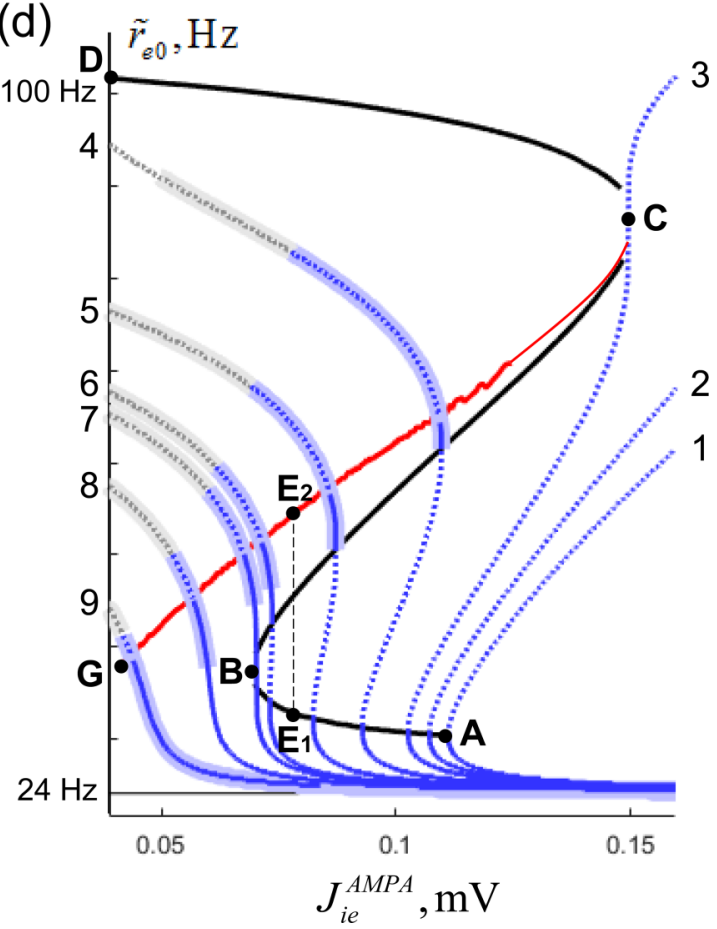

FIG. 8. Bifurcation diagram of the time-averaged forced system (a), (b). Horizontal axis, $J_{i e}^{\mathrm{AMPA}}$; vertical axis, $J_{i e}^{\mathrm{NMDA}}$. Black line ABCD, saddle-node bifurcations; $\mathrm{B}$ and $\mathrm{C}$ are the cusp points. Red line CG, Hopf bifurcations. Green line, focus-to-node transitions. The point $\mathrm{E}$ is the intersection between $\mathrm{CG}$ and $\mathrm{AB}$. In the region to the left of the blue line, the unforced system is unstable. Horizontal lines, the probed values of $J_{i e}^{\mathrm{NMDA}}$; they are denoted by the numbers 1-9 at the right parts of the panels. (c), (d) Blue/gray lines, 1D bifurcation diagrams (dependence of the time-averaged forced $\tilde{r}_{e}$ equilibria on $J_{i e}^{\mathrm{AMPA}}$ ) for various values of $J_{i e}^{\mathrm{NMDA}}$. Diagrams are marked by the numbers that correspond to the horizontal lines in panels (a) and (b). Solid lines, an equilibrium is stable; dashed lines, unstable. Blue color, the unforced equilibrium is stable; gray color, unstable. Thin lines only, an equilibrium is a node or a saddle; superimposed thick lines, an equilibrium is a focus. Red line CG connects all Hopf bifurcation points; black line ABCD connects all saddle-node points; the meaning of A, B, C, D, G is the same as in panel (a). $E_{1}$ and $E_{2}$ correspond to the point $E$ in (a). The numbers 1-9 in the left and the right parts of the panels link the 1D diagrams in panels (c) and (d) with the selected $J_{i e}^{\mathrm{NMDA}}$ levels (horizontal lines) in (a) and (b). The thin segment of the red line was drawn manually (see Appendix C for details). 
To explore relations between points on the $2 \mathrm{D}$ bifurcation diagram and the oscillation-induced shift $\Delta r_{e 0}$, we selected several representative values of $J_{i e}^{\mathrm{NMDA}}$ [denoted by horizontal lines and marked by numbers in Figs. 8(a) and 8(b)]. For each of these $J_{i e}^{\mathrm{NMDA}}$ values, we plotted the $\tilde{r}_{e}$ coordinates of the fixed points (i.e., the equilibrium time-averaged excitatory firing rates) versus $J_{i e}^{\mathrm{AMPA}}$ values, thus obtaining onedimensional (1D) bifurcation diagrams of the time-averaged forced system. These diagrams are shown in Fig. 8(c) by the blue or gray lines, and marked by the same numbers as the $J_{i e}^{\mathrm{NMDA}}$ levels in Figs. 8(a) and 8(b); a region of interest is shown in more detail in Fig. 8(d). Stable parts of the diagrams in Figs. 8(c) and 8(d) are represented by solid lines, and unstable parts are represented by dashed lines. Gray parts correspond to unstable behavior of the unforced system. Points at thin parts of the diagrams are nodes or saddles, while points at the thick parts are foci. All points of saddle-node bifurcations are connected by the black line, and all points of Hopf bifurcations are connected by the red line. The points A, B, C, D, G have the same meaning as in Figs. 8(a) and 8(b); the points $E_{1}$ and $E_{2}$ correspond to the same point $E$ in Figs. 8(a) and $8(\mathrm{~b})$. The unforced firing rate $r_{e 0}=24 \mathrm{~Hz}$ is represented by a horizontal line in the bottom part of Figs. 8(c) and 8(d). Thus, for a point on a $1 \mathrm{D}$ diagram, the oscillation-induced firing rate shift $\Delta r_{e 0}$ is a vertical distance from this point to the $r_{e 0}$ line mentioned above.

Now we will go through the selected $J_{i e}^{\mathrm{NMDA}}$ levels (1-9) and discuss the properties of the corresponding 1D diagrams that determine the maximal achievable $\tilde{r}_{e 0}$ (and, thus, the maximal $\Delta r_{e 0}$ ).

For all $J_{i e}^{\text {NMDA }}$ values below the cusp point B [lines 1-6 in Fig. 8(a)], the corresponding 1D diagrams contain a stable branch that terminates by a saddle-node bifurcation [see the lowest parts of diagrams $1-6$ in Figs. 8(c) and 8(d) that are located below the black line AB]. For $J_{i e}^{\mathrm{NMDA}}$ values below the cusp point $C$ [lines 1 and 2 in Fig. 8(a)], this is the only stable branch of a 1D diagram. Consequently, the maximal values of $\tilde{r}_{e 0}$ for diagrams 1 and 2 in Figs. 8(c) and 8(d) lie on the black line $\mathrm{AB}$, and the corresponding $\Delta r_{e 0}$ values are small.

For $J_{i e}^{\mathrm{NMDA}}$ values between the cusp points $\mathrm{C}$ and $\mathrm{B}$ [lines 4-6 in Fig. 8(a)], the corresponding 1D diagrams contain, in addition to the lower stable branch (below AB), another branch with higher $\tilde{r}_{e}$, part of which is stable. In Figs. 8(c) and $8(\mathrm{~d})$, these stable parts are located between the black line $\mathrm{BC}$ and the red line $\mathrm{CG}$.

For $J_{i e}^{\text {NMDA }}$ values between the points $\mathrm{C}$ and $\mathrm{E}$ [lines 4 and 5 in Fig. 8(a)], the Hopf bifurcation (crossing of the red line $\mathrm{CG}$ ) occurs at higher values of $J_{i e}^{\mathrm{AMPA}}$ than the saddle-node bifurcation (crossing of the black line $\mathrm{AB}$ ). Thus, for any stable point on the upper branch of a 1D diagram in Figs. 8(c) and 8(d) (between CG and BC) there exists a point on the lower stable branch of the same diagram (below AB). However, the probability that the system will settle down on the upper branch is very low, so we could still assume that maximal $\tilde{r}_{e 0}$ values are located on the $\mathrm{AB}$ line, and $\Delta r_{e 0}$ are small.

For $J_{i e}^{\mathrm{NMDA}}$ values between the points $\mathrm{E}$ and $\mathrm{B}$ [line 6 in Fig. 8(a)], the Hopf bifurcation occurs at lower values of $J_{i e}^{\text {AMPA }}$ than the saddle-node bifurcation. Thus, for any of these $J_{i e}^{\mathrm{NMDA}}$ values, there exists a small $J_{i e}^{\mathrm{AMPA}}$ range [between the
CG and BC lines in Fig. 8(a)], for which all stable solutions $\tilde{r}_{e 0}$ lie on the upper branch of a corresponding 1D diagram. Consequently, the maximal $\tilde{r}_{e 0}$ values lie on the CG line in Fig. 8(d), providing high $\Delta r_{e 0}$ values.

For $J_{i e}^{\text {NMDA }}$ values above the cusp point C [lines 8 and 9 in Fig. 8(a)], the upper and lower branches of 1D diagrams are merged into a single stable branch [see diagrams 8 and 9 in Figs. 8(c) and 8(d)]; this branch loses stability via Hopf bifurcation as $J_{i e}^{\text {AMPA }}$ decreases (crossing the red line $\mathrm{CG}$ ). Consequently, the maximal achievable $\tilde{r}_{e 0}$ values are, again, located on the CG line, so relatively high $\Delta r_{e 0}$ values could be reached.

In summary, the maximal mean firing rate values $\tilde{r}_{e 0}$ that could be achieved in the forced system under various combinations of $J_{i e}^{\mathrm{NMDA}}$ and $J_{i e}^{\mathrm{AMPA}}$ are located on the two distinct curves on the $\left(\tilde{r}_{e}, J_{i e}^{\mathrm{AMPA}}\right)$ plane [depicted in Fig. 8(d)]. For small $J_{i e}^{\mathrm{NMDA}}$ values, the maximal $\tilde{r}_{e 0}$ values lie on the $\mathrm{AE}_{1}$ curve (so they are small); for large $J_{i e}^{\mathrm{NMDA}}$ values, they lie on the $\mathrm{E}_{2} \mathrm{G}$ curve (and thus could be large). The maximal $\tilde{r}_{e 0}$ over all combinations of $J_{i e}^{\mathrm{NMDA}}, J_{i e}^{\mathrm{AMPA}}$ is given by the point $\mathrm{E}_{2}$; the corresponding oscillation-induced firing rate shift $\Delta r_{e 0}$ equals to approximately $20 \mathrm{~Hz}$. We should note that achieving this shift requires very fine-tuned weights. For larger $J_{i e}^{\mathrm{NMDA}}$ values, the maximal achievable $\Delta r_{e 0}$ values are somewhat smaller [see diagrams 8 and 9 in Fig. 8(d)], but more robust to parameter variations.

\section{DISCUSSION AND CONCLUSION}

Numerous theoretical concepts of neural processing (e.g., selectivity to certain information) are formulated in terms of average firing rates [2]. At the same time, brain activity demonstrates collective oscillatory patterns that correlate with functional states, task requirements, and behavioral features. In order to build a theory that reconciles rate-based neural coding with the functional role of oscillations in computations and information routing, one should consider a nonlinear mechanism that converts oscillatory power into tonic firing rate shifts $[5,10]$. In this paper, we explored a potential role that NMDA receptors, which are known to have nonlinear behavior, could play in shifting the average population activity level in the presence of an external oscillatory input. We considered a system composed of an excitatory and an inhibitory population, described by a mean-field model with population firing rates, mean membrane voltages, and NMDA currents as the dynamical variables. In order to delineate the NMDArelated effects from the effects of nonlinearity of neural gain functions, we linearized the dependences of the firing rate and the mean voltage on the synaptic input about the unperturbed equilibrium of the system.

Our main finding is that strong recurrent excitatory-toexcitatory NMDA coupling produces positive oscillationinduced firing rate shifts, while additional weak excitatoryto-inhibitory NMDA coupling stabilizes them. Both effects are mediated by the interactions between the excitatory firing rate oscillations and the membrane voltage oscillations via the mechanisms based on the specific properties of NMDA receptors. In the appropriate parameter region, considerable stable shifts (about $10 \mathrm{~Hz}$ ) could be achieved. 
The result in large part stems from the NMDA receptors' unique nonlinear property that distinguishes them from other synaptic receptor types. An ion channel associated with an NMDA receptor is blocked by a magnesium ion when the neuron is at rest and the membrane voltage near the receptor is hyperpolarized (typically -70 to $-80 \mathrm{mV}$ ). The probability that the channel is freed from this block increases nonlinearly with depolarization of the postsynaptic membrane voltage [see the expression (3)]. Thus, an NMDA channel opens when (a) a neurotransmitter molecule released by a presynaptic neuron when it fires a spike binds to the receptor and (b) the channel is free from the magnesium block. Consequently, the proportion of opened NMDA channels increases both with the presynaptic firing rate and the postsynaptic membrane voltage. The average population NMDA activity in our model depends on the product of two terms: (a) firing rate of the excitatory population and (b) a nonlinear function of the mean membrane voltage of the population that receives the NMDA input. Hence, the NMDA-receptor mediated coupling is dependent jointly on the presynaptic and the postsynaptic neural activity.

We delivered a zero-mean sinusoidal input to our system, which entrained oscillations of the firing rate and of the membrane voltage in both populations. Using the fact that NMDA dynamics are slow, we applied time-scale separation and time-averaging methods to analyze the periodically forced system. For each of the populations, we obtained additional terms that enter the NMDA dynamics and reflect the timeaveraged effect of the oscillations [see (16)]. The first term reflected the joint effect of two sinusoidal signals: (a) the firing rate oscillations of the excitatory population and (b) the membrane voltage oscillations of the considered population. If these signals are not orthogonal, the time average of their product is nonzero. The second term reflected the effect of the sinusoidal membrane voltage oscillations fed into the nonlinear function (the voltage dependence of the magnesium block). The resulting signal is itself nonlinear, so its time average is, again, nonzero. We found that the first term largely dominated the second one, because the phase lags between the firing rate and the voltage signals were small (see Table II in Appendix A1; $\cos \varphi_{e}$ and $\cos \varphi_{i}$ are close to 1 ), while the voltage dependence of the magnesium block is close to linear in the physiological range of voltages.

Based on the aforementioned results, we developed a geometrical method of finding steady states of the unforced system and of the time-averaged forced system. To tease apart the complementary roles played by the excitatory-toexcitatory and the excitatory-to-inhibitory NMDA coupling, we applied our method to two models. In model 1, the NMDA receptors were located exclusively on the excitatory neurons, while in model 2 they were located both on the excitatory and on the inhibitory neurons. For model 1, we showed geometrically that the oscillation-induced mean firing rate shift is strongly limited by stability requirements (Fig. 1). Adding even a very weak NMDA coupling between the excitatory and the inhibitory population (model 2) allowed us to overcome these limitations and achieve pronounced firing rate shift (up to $10 \mathrm{~Hz}$ ) without destabilizing the system (Fig. 3). We confirmed the predictions of the geometrical analysis by direct numerical simulation of the models (Figs. 2 and 4). We also simulated model 2 with different NMDA time constants (Fig. 5). We found that the oscillation-induced rise of the average firing rate is steeper when the NMDA time constant is smaller [Figs. 5(a) and 5(b)]. We also found that the resulting average firing rate shifts differ from the predictions towards larger values more strongly for small NMDA time constants [Figs. 5(a) and 5(b)], but the prediction is almost perfect for very large time constants [Fig. 5(d)]. Finally, we explored the parameter space by varying synaptic weights and found optimal regions of parameters, in which the strongest mean firing rate shift could be achieved under the same amplitude of the entrained oscillations.

The NMDA-based mechanism we proposed has one major advantage: it could provide a considerable firing rate shift by entraining oscillations with important features present in real cortical networks and conceptually described as sparse synchrony [1]. The shape of such experimentally observed oscillations is close to sinusoidal and their amplitude is relatively small compared to the baseline firing rate, which is true in our model (see Figs. 2 and 4 and Appendix A5). Sparse synchrony in spiking networks is observed only at the population level, while individual neurons stay in the subthreshold regime and generate Poisson-like spike trains driven by noise. We confirmed that the steady state of the unforced system corresponds to Poisson-like activity and that the voltage oscillations do not cause crossing of the spikegeneration threshold (see Appendix A5). We note that, in principle, zero-mean sinusoidal input could induce firing rate shifts due to nonlinearity of neural gain functions. However, obtaining a pronounced firing rate shift on a basis of neural gain functions' nonlinearity only would require strong induced oscillations with amplitudes comparable to the baseline firing rate and rectified nonsinusoidal shape.

We should note that we did not aim to explore frequency dependence of the time-averaged equilibrium shift induced by input oscillations. Our goal was to demonstrate that a pronounced shift could be achieved via a NMDA-based mechanism. Nevertheless, let us outline several mechanisms that could account for the frequency dependence of the shift in extended models. First, in our approach, we kept the entrained amplitudes constant for any set of parameters by readjusting the input amplitudes. Without this readjustment, the entrained amplitudes would depend on the input frequency (for given input amplitudes), so the equilibrium shift would be also frequency dependent. Second, we applied the slow-fast approximation in our analysis, assuming that the slow subsystem (composed of the NMDA currents) does not oscillate. In fact, slow input oscillations would entrain the slow subsystem, making the resulting effect frequency dependent. Third, we used a weakly nonlinear approach, in which the shift depends only on the amplitude of the entrained oscillations, but not on its frequency. In fact, oscillations could interact with inherent strongly nonlinear behavior of the system (i.e., with state switching in bistable systems), making the oscillation-induced equilibrium shift to be frequency dependent (see Dipoppa and Gutkin [7] and Schmidt et al. [9]). Detailed exploration of these mechanisms is a matter of future research.

There are two main issues related to our results. First, strong shifts $(10-20 \mathrm{~Hz})$ in our model required parameter tuning [e.g., the yellow regions in Figs. 6(a), 7(a), and 7(b)]. 
However, moderate shifts (about $5 \mathrm{~Hz}$ ) could be achieved in a relatively wide parameter region. Several empirical studies (e.g., $[17,18]$ ) demonstrate that the firing rate shifts observed in the presence of oscillations fall into this moderate range of values. Secondly, the oscillation-induced firing rate shift builds up very slowly; for example, the transition in Fig. 4 takes about $5 \mathrm{~s}$ to develop. Such slow effects could play a role at the level of brain functional states (i.e., rest vs vigilance), but they are not well suited for fast cognitive operations (i.e., perceptual decision making). Nevertheless, as the maximal firing rate shift in our model is large, a more realistic smaller shift could be reached faster (i.e., the maximal shift in Fig. 4 is $13 \mathrm{~Hz}$, but a $3-\mathrm{Hz}$ shift could be reached already after $750 \mathrm{~ms}$ of oscillatory input). While shifts in the $\mathrm{Hz}$ range may seem limited, experiments on cognitive tasks have found that such shifts indeed specifically distinguish activity related to task performance vs task-irrelevant firing (e.g., item-coding working memory activity [19]). Furthermore, we demonstrated that decreased (but still realistic) values of the NMDA time constant yield much faster rise of the mean firing rate [Figs. 5(a) and 5(b)]. For example, with the NMDA time constant of $50 \mathrm{~ms}$, a $5-\mathrm{Hz}$ shift could be reached after $265 \mathrm{~ms}$ of oscillatory input, which is comparable to the time scale of fast neural processes such as sensory discrimination.

We should now point out several limitations of our analysis. First, gain functions of real neurons are nonlinear, so firing rate shifts are affected both by gain function nonlinearities and by the NMDA-related mechanism discussed here. However, in a realistic asynchronous irregular regime, the gain function nonlinearities are weak, so we suggest that their effect would not be very pronounced. The next limitation is related to the fact that our model of NMDA activity is current based (i.e., activity of the channels is interpreted as an input current to a neuron). In fact, the channel activity changes the membrane conductance, and the input current is calculated by Ohm's law, where the driving force is the difference between the membrane voltage and the constant reversal potential of the channels. Thus, the membrane voltage affects not only the coefficient related to the magnesium block but also the driving force. We expect that the resulting oscillation-induced firing rate shifts would be somewhat smaller in this case than predicted by our analysis. Furthermore, real neurons may have long dendritic trees, and spikes generated by these neurons back-propagate to the dendritic tree, affecting membrane voltages at synaptic sites. Thus, under periodic forcing, the expression for the voltage amplitude would contain an additional term corresponding to the firing rate oscillations filtered by dendritic compartments. This could potentially produce various effects not accounted for by our model. Finally, in this paper, we explored only the case of positive oscillation-induced firing rate shifts. They are based on strong excitatory-to excitatory NMDA connections (which cause the shift) and weak excitatory-to-inhibitory NMDA connections (which stabilize the shift). The case of strong excitatory-toinhibitory NMDA connections (which, in fact, exist in the cortex) causing negative oscillation-induced shifts is beyond the scope of this paper. All the aforementioned issues are promising lines of future investigations.

The main principle of the proposed NMDA-based mechanism could be generalized. A system demonstrating oscillation-induced firing rate shift should contain a variable the dynamics of which nonlinearly depends on a fast variable or on a combination of fast variables. In our case, such variable is NMDA current; as we demonstrated, its dynamics approximately depends on the product of the population firing rate and the membrane voltages (both of which are fast), so this dependency is nonlinear. A dependent variable does not necessarily need to be slow, but if it is (as in our case) this simplifies the analysis. The variables that enter the nonlinear expression should be fast because otherwise they would not be entrained by input oscillations, and thus would not be able to mediate the effect of these oscillations on the average firing rate. As an example, we could consider a short-term plasticity governed by the Tsodyks-Markram rule [20]. This rule is, in general, nonlinear; however, it is linear by the only fast variable it depends on (the population firing rate), and the two other variables (which describe the plasticity) are slow. Thus, such type of short-term plasticity could not act as a mechanism of oscillation-induced firing rate shift.

In summary, it is clear that many neural processes could potentially link oscillatory activity to mean firing rate modulations. Besides the nonlinearity of the spike generation, one could propose mechanisms based on nonlinear behavior of various slow voltage-dependent ion channels, transitions between spiking and bursting, activity of metabotropic receptors, or certain synaptic plasticity types. Furthermore, the character of such link should critically depend on microconnectivity patterns. Nevertheless, the NMDA-based mechanism that we proposed in this paper is rather general and should play a certain role in oscillatory-induced firing rate shifts (alongside other potential mechanisms) in most neural configurations. However, the extent to which the NMDArelated nonlinearity is involved in these shifts in various tasks and functional states is a subject of future experimental research.

\section{ACKNOWLEDGMENT}

This article was prepared within the framework of the HSE University Basic Research Program and funded by the Russian Academic Excellence Project No. 5-100.

\section{APPENDIX A: PARAMETER SELECTION}

\section{Primary parameters}

In Table I, the parameters with a priori selected values are presented. We used typical values for the parameters of individual neurons $\left(\tau_{m e}, \tau_{m i}, g_{m e}, g_{m i}, V_{e}^{r}, V_{i}^{r}, V_{e}^{\text {th }}, V_{i}^{\text {th }}, E_{L e}, E_{L i}\right)$, numbers of inputs $\left(K_{e}, K_{i}\right)$, and NMDA time constant $\left(\tau_{\mathrm{NMDA}}\right)$. The fixed point was set by total mean inputs $u_{e 0}, u_{i 0}$ and total input variances $\sigma_{e 0}^{2}, \sigma_{i 0}^{2}$; such inputs provided reasonable firing rates $r_{e 0}, r_{i 0}$ and CV's [CV is the coefficient of interspike interval (ISI) variability, defined as standard deviation of ISI divided by mean ISI]. See Appendix A 5, for more details. We also preselected the amplitudes $\left|r_{e}^{A}\right|,\left|r_{i}^{A}\right|$ of the forced oscillations and chose the zero lag $\gamma_{r}=0$ between them.

During the selection of the synaptic weights, we used the same phase plane visualization method as for the main experiments. We exploited the convenient property of our 
TABLE I. Primary parameters.

\begin{tabular}{|c|c|c|c|c|c|}
\hline \multicolumn{6}{|c|}{ Common parameters } \\
\hline Parameter & Value & Parameter & Value & Parameter & Value \\
\hline$K_{e}$ & 800 & $\tau_{m e}$ & $20 \times 10^{-3} \mathrm{~s}$ & $u_{e 0}$ & $-67.25 \times 10^{-3} \mathrm{~V}$ \\
\hline$K_{i}$ & 200 & $\tau_{m i}$ & $10 \times 10^{-3} \mathrm{~s}$ & $u_{i 0}$ & $-65.25 \times 10^{-3} \mathrm{~V}$ \\
\hline$\tau_{\mathrm{NMDA}}$ & $200 \times 10^{-3} \mathrm{~s}$ & $g_{m e}=g_{m i}$ & $1 \mathrm{~S} / \mathrm{m}^{2}$ & $\sigma_{e 0}$ & $14.2 \times 10^{-3} \mathrm{~V}$ \\
\hline$j_{e e}^{\mathrm{AMPA}}$ & $0 \mathrm{~V}$ & $V_{e}^{\mathrm{r}}=V_{i}^{\mathrm{r}}$ & $-70 \times 10^{-3} \mathrm{~V}$ & $\sigma_{i 0}$ & $13 \times 10^{-3} \mathrm{~V}$ \\
\hline$j_{e e}^{\mathrm{NMDA}}$ & $0.15 \times 10^{-2} \mathrm{~A} / \mathrm{m}^{2}$ & $V_{e}^{\mathrm{th}}=V_{i}^{\mathrm{th}}$ & $-55 \times 10^{-3} \mathrm{~V}$ & $\omega / 2 \pi$ & $20 \mathrm{~Hz}$ \\
\hline$j_{e i}$ & $-1 \times 10^{-3} \mathrm{~V}$ & $E_{L e}=E_{L i}$ & $-70 \times 10^{-3} \mathrm{~V}$ & $\left|r_{e}^{A}\right|$ & $10 \mathrm{~Hz}$ \\
\hline$j_{i i}$ & $0 \mathrm{~V}$ & $\gamma_{r}$ & 0 & $\left|r_{i}^{A}\right|$ & $5 \mathrm{~Hz}$ \\
\hline \multicolumn{2}{|c|}{ Model 1} & \multicolumn{2}{|c|}{ Model 2} & & \\
\hline Parameter & Value & Parameter & Value & & \\
\hline$j_{i e}^{\mathrm{AMPA}}$ & $0.117 \times 10^{-3} \mathrm{~V}$ & $j_{i e}^{\mathrm{AMPA}}$ & $0.06 \times 10^{-3} \mathrm{~V}$ & & \\
\hline$j_{i e}^{\mathrm{NMDA}}$ & $0 \mathrm{~A} / \mathrm{m}^{2}$ & $j_{i e}^{\mathrm{NMDA}}$ & $0.003 \times 10^{-2} \mathrm{~A} / \mathrm{m}^{2}$ & & \\
\hline
\end{tabular}

system that the $\tilde{I}_{\text {NMDAe }}$ curve does not depend on the fast synaptic weights. A change of the fast weights caused a "rotation" of the $\tilde{r}_{e}$ curve around the unforced equilibrium $\left(r_{e 0}, I_{\mathrm{NMDA} e 0}\right)$, not affecting the $\tilde{I}_{\mathrm{NMDA} e}$ curve, so the position of the time-averaged forced equilibrium $\left(\tilde{r}_{e 0}, \tilde{I}_{\mathrm{NMDA} e 0}\right)$ was easy to predict. Thus, we were able to control the fixed point of the time-averaged forced system by changing the fast synaptic weights (at the same time automatically adjusting the external inputs to keep the unforced equilibrium unchanged).
In Appendices A2-A4, we derive the population time constants, the gain function slopes, and the parameters of the external input from the primary parameters, using analytical methods, as well as numerical simulations of individual LIF neurons and neural populations. The derived parameters are presented in Table II. In Appendix A5, we determine several properties of our model behavior and test whether they are biologically plausible.

TABLE II. Derived parameters and characteristics of equilibria.

\begin{tabular}{|c|c|c|c|c|c|}
\hline \multicolumn{6}{|c|}{ Common parameters } \\
\hline Parameter & Value & Parameter & Value & Parameter & Value \\
\hline$r_{e 0}$ & $24.2 \mathrm{~Hz}$ & $V_{e 0}$ & $-75.1 \times 10^{-3} \mathrm{~V}$ & $C V_{e 0}$ & 1.1 \\
\hline$r_{i 0}$ & $29.5 \mathrm{~Hz}$ & $V_{i 0}$ & $-69.9 \times 10^{-3} \mathrm{~V}$ & $C V_{i 0}$ & 0.9 \\
\hline$c_{r e}$ & 1921.2 & $\tau_{r e}$ & $6.2 \times 10^{-3} \mathrm{~s}$ & $\left|V_{e}^{A}\right|$ & $2.4 \times 10^{-3} \mathrm{~V}$ \\
\hline$c_{r i}$ & 3528.9 & $\tau_{r i}$ & $2.8 \times 10^{-3} \mathrm{~s}$ & $\left|V_{i}^{A}\right|$ & $0.6 \times 10^{-3} \mathrm{~V}$ \\
\hline$c_{V e}$ & 0.506 & $\tau_{V e}$ & $7.9 \times 10^{-3} \mathrm{~s}$ & $\cos \varphi_{e}$ & 0.993 \\
\hline$c_{V i}$ & 0.454 & $\tau_{V i}$ & $4.4 \times 10^{-3} \mathrm{~s}$ & $\cos \varphi_{i}$ & 0.986 \\
\hline \multicolumn{6}{|c|}{ Model 1} \\
\hline Parameter & Value & Parameter & Value & Parameter & Value \\
\hline$h_{x e}$ & $-70.3 \times 10^{-3} \mathrm{~V}$ & $\sigma_{x e}$ & $11.9 \times 10^{-3} \mathrm{~V}$ & $I_{\mathrm{NMDA} e 0}$ & $\overline{19.1 \times 10^{-2} \mathrm{~A} / \mathrm{m}^{2}}$ \\
\hline$h_{x i}$ & $-17.9 \times 10^{-3} \mathrm{~V}$ & $\sigma_{x i}$ & $9.1 \times 10^{-3} \mathrm{~V}$ & $I_{\text {NMDA } i 0}$ & $0 \mathrm{~A} / \mathrm{m}^{2}$ \\
\hline$\left|h_{e}^{A}\right|$ & $25.5 \times 10^{-3} \mathrm{~V}$ & $\arg h_{e}^{A}$ & 0.16 & & \\
\hline$\left|h_{i}^{A}\right|$ & $8.0 \times 10^{-3} \mathrm{~V}$ & $\arg h_{i}^{A}$ & 3.08 & & \\
\hline$\tilde{r}_{e 0}$ & $27.1 \mathrm{~Hz}$ & $\tilde{\tilde{V}}_{e 0}$ & $-74.3 \times 10^{-3} \mathrm{~V}$ & $\tilde{I}_{\mathrm{NMDA} e 0}$ & $23.1 \times 10^{-2} \mathrm{~A} / \mathrm{m}^{2}$ \\
\hline$\tilde{r}_{i 0}$ & $39.0 \mathrm{~Hz}$ & $\tilde{V}_{i 0}$ & $-68.7 \times 10^{-3} \mathrm{~V}$ & $\tilde{I}_{\mathrm{NMDA} i 0}$ & $0 \mathrm{~A} / \mathrm{m}^{2}$ \\
\hline \multicolumn{6}{|c|}{ Model 2} \\
\hline Parameter & Value & Parameter & Value & Parameter & Value \\
\hline$h_{x e}$ & $-70.3 \times 10^{-3} \mathrm{~V}$ & $\sigma_{x e}$ & $11.9 \times 10^{-3} \mathrm{~V}$ & $I_{\mathrm{NMDA} e 0}$ & $19.1 \times 10^{-2} \mathrm{~A} / \mathrm{m}^{2}$ \\
\hline$h_{x i}$ & $-12.0 \times 10^{-3} \mathrm{~V}$ & $\sigma_{x i}$ & $9.2 \times 10^{-3} \mathrm{~V}$ & $I_{\mathrm{NMDA} i 0}$ & $0.5 \times 10^{-2} \mathrm{~A} / \mathrm{m}^{2}$ \\
\hline$\left|h_{e}^{A}\right|$ & $25.5 \times 10^{-3} \mathrm{~V}$ & $\arg h_{e}^{A}$ & 0.16 & & \\
\hline$\left|h_{i}^{A}\right|$ & $3.4 \times 10^{-3} \mathrm{~V}$ & $\arg h_{i}^{A}$ & 3.00 & & \\
\hline$\tilde{r}_{e 0}$ & $36.9 \mathrm{~Hz}$ & $\tilde{V}_{e 0}$ & $-71.7 \times 10^{-3} \mathrm{~V}$ & $\tilde{I}_{\mathrm{NMDA} e 0}$ & $36.3 \times 10^{-2} \mathrm{~A} / \mathrm{m}^{2}$ \\
\hline$\tilde{r}_{i 0}$ & $71.0 \mathrm{~Hz}$ & $\tilde{V}_{i 0}$ & $-64.6 \times 10^{-3} \mathrm{~V}$ & $\tilde{I}_{\mathrm{NMDA} i 0}$ & $1.1 \times 10^{-2} \mathrm{~A} / \mathrm{m}^{2}$ \\
\hline
\end{tabular}


(a) 35

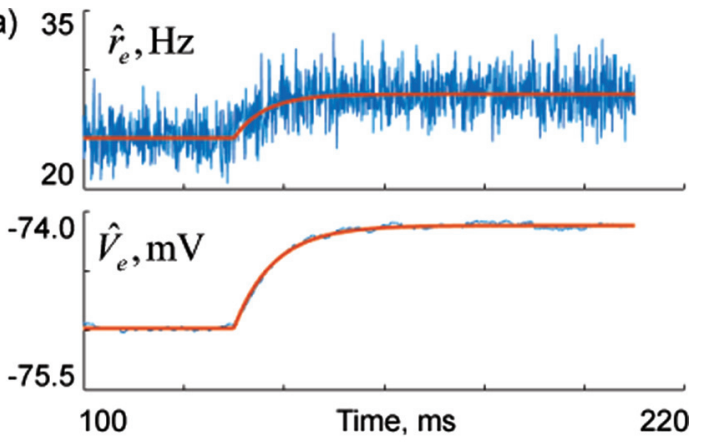

(b)
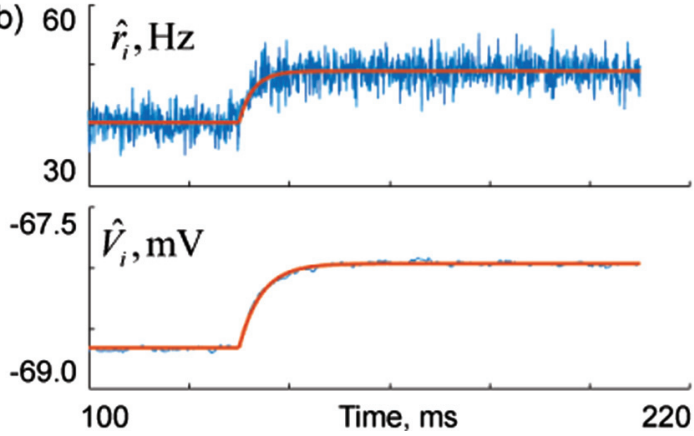

FIG. 9. Results of simulation of an uncoupled population of excitatory (a) and inhibitory (b) neurons. Top panels, dynamics of the population firing rate; bottom panels, dynamics of the mean membrane voltage. Blue lines, simulation results; red lines, exponential fits.

\section{Linearization coefficients and $\mathrm{CV}$ values}

In our numerical computations, we assumed that the input variance does not depend on the state of the system (which is a reasonable assumption if fast synaptic coupling is sufficiently weak). Following this assumption, we simulated a single excitatory (and a single inhibitory) leaky integrate-and-fire neuron that received Gaussian white noise with the amplitude $\sigma_{a 0}^{2}$ and the tonic input $u_{a}$. We probed several values of $u_{a}$ around $u_{a 0}$. The simulations were governed by the following equations:

$$
\begin{aligned}
\tau_{m a} \frac{d v_{a}}{d t} & =-v_{a}+E_{L a}+u_{a}+\sigma_{a 0} \eta_{a}(t) \\
v_{a} & >V_{a}^{\mathrm{th}}: v_{a} \leftarrow V_{a}^{r},
\end{aligned}
$$

where $v_{a}$ is the membrane voltage, $\tau_{m a}$ is the membrane time constant, $E_{L a}$ is the resting potential, $\eta_{a}(t)$ is white noise with zero mean and unit standard deviation, $V_{a}^{\text {th }}$ is the spiking threshold, and $V_{a}^{r}$ is the reset voltage.

For each value of $u_{a}$, we calculated the resulting mean membrane voltage $\hat{V}_{a}^{s s, 0}\left(u_{a}\right)$ and the firing rate $\hat{r}_{a}^{s s, 0}\left(u_{a}\right)$. Given these empirical dependencies, we numerically estimated their derivatives at $u_{a 0}$, thus determining the constants $c_{r a}$ and $c_{V a}$. Also, from the simulated spike train for $u_{a}=u_{a 0}$ we calculated the coefficient of interspike interval variation $C V_{a 0}$ which characterizes irregularity of the spike train.

\section{Population time constants}

In order to find population time constants $\tau_{r a}$ and $\tau_{V a}$, we considered $N=5000$ uncoupled neurons the dynamics of which are governed by (A1) with $u_{a}=u_{a 0}$. We repeated this simulation 20 times. For each time bin, we averaged the membrane voltages over neurons and trials, thus computing the empirical temporal dynamics of the population membrane voltage $\hat{V}_{a}(t)$. We also averaged the numbers of spikes produced by the network over trials, thus computing the empirical temporal dynamics of the population firing rate $\hat{r}_{a}(t)$. At a time moment when $\hat{r}_{a}(t)$ and $\hat{V}_{a}(t)$ were already stabilized (given $u_{a}=u_{a 0}$ ), we increased $u_{a}$ by $\Delta u=2 \mathrm{mV}$, continued the simulations, and calculated $\hat{r}_{a}(t)$ and $\hat{V}_{a}(t)$ for the subsequent time moments, until they stabilized again. Then we fitted exponential functions to the transitions of $\hat{r}_{a}(t)$ and $\hat{V}_{a}(t)$ produced by the increase of $u_{a}$, from which we got $\tau_{r a}$ and $\tau_{V a}$, respectively. The resulting $\hat{r}_{a}(t)$ and $\hat{V}_{a}(t)$ for the excitatory and the inhibitory network (and the corresponding exponential fits) are presented in Figs. 9(a) and 9(b), respectively.

\section{Parameters of the external inputs}

Using the single-neuron simulation (as in the previous paragraphs), we numerically defined the steady-state population firing rate $r_{a 0}$ and the population membrane voltage $V_{a 0}$ given the input $u_{a}=u_{a 0}$ :

$$
\begin{aligned}
r_{a 0} & =\hat{r}_{a}^{s s, 0}\left(u_{a 0}\right) \\
V_{a 0} & =\hat{V}_{a}^{s s, 0}\left(u_{a 0}\right) .
\end{aligned}
$$

From the found values of $r_{a 0}$ and $V_{a 0}$, using (2), we calculated the steady-state NMDA currents $I_{\text {NMDA } a 0}$ :

$$
I_{\mathrm{NMDA} a 0}=I_{\mathrm{NMDA} a}^{s s}\left(r_{e 0}, V_{a 0}\right) .
$$

Then we determined the mean total inputs $h_{e}, h_{i}$; the mean external inputs $h_{x e}, h_{x i}$; and the external input variances $\sigma_{x e}^{2}, \sigma_{x i}^{2}$ :

$$
\begin{aligned}
h_{e} & =u_{e 0}-\left(j_{e e}^{\mathrm{AMPA}} K_{e e} \tau_{m e} r_{e 0}-j_{e i} K_{e i} \tau_{m e} r_{i 0}+I_{\mathrm{NMDAe} 0} / g_{m e}\right) \\
h_{i} & =u_{i 0}-\left(j_{i e}^{\mathrm{AMPA}} K_{i e} \tau_{m i} r_{e 0}-j_{i i} K_{i i} \tau_{m i} r_{i 0}+I_{\mathrm{NMDA} 0} / g_{m i}\right) \\
h_{x e} & =h_{e}-E_{L e} \\
h_{x i} & =h_{i}-E_{L i} \\
\sigma_{x e}^{2} & =\sigma_{e 0}^{2}-\frac{1}{2}\left(\left(j_{e e}^{\mathrm{AMPA}}\right)^{2} K_{e e} \tau_{m e} r_{e 0}+j_{e i}^{2} K_{e i} \tau_{m e} r_{i 0}\right) \\
\sigma_{x i}^{2} & =\sigma_{i 0}^{2}-\frac{1}{2}\left(\left(j_{i e}^{\mathrm{AMPA}}\right)^{2} K_{i e} \tau_{m i} r_{e 0}+j_{i i}^{2} K_{i i} \tau_{m i} r_{i 0}\right) .
\end{aligned}
$$

Although $h_{x e}, h_{x i}, \sigma_{x e}^{2}, \sigma_{x i}^{2}$ do not participate directly in our analysis, we use them to check whether these values could be achieved under realistic conditions.

In order to calculate the external oscillatory inputs, we used the real-valued amplitudes $\left|r_{e}^{A}\right|,\left|r_{i}^{A}\right|$ of the forced oscillations delivered to the excitatory and inhibitory populations, respectively, as well as the phase lag $\gamma_{r}$ between them, and constructed the corresponding complex-valued amplitudes as follows:

$$
\begin{aligned}
& r_{e}^{A}=\left|r_{e}^{A}\right| \\
& r_{i}^{A}=\left|r_{i}^{A}\right| \exp \left(i \gamma_{r}\right) .
\end{aligned}
$$

Then we calculated the complex amplitudes $h_{e}^{A}, h_{i}^{A}$ of the oscillatory external inputs using the expression (10). 


\section{Parameter validation}

Here we demonstrate that the parameters we selected are physiologically plausible and lead to realistic behavior of the system.

Postsynaptic potentials produced by activation of the fast receptors are given by the synaptic weights $j_{e e}^{\mathrm{AMPA}}, j_{i e}^{\mathrm{AMPA}}, j_{e i}, j_{i i}$ and provided in Table I. Postsynaptic potentials produced by the NMDA receptors' activation were equal to $1.16 \mathrm{mV}$ for the E-E connections and to $0.026 \mathrm{mV}$ for the E-I connection. All postsynaptic potentials lie in a physiological range roughly below $1.5 \mathrm{mV}$.

In our model, we defined the tonic external inputs by their mean values $h_{x a}$ and standard deviations $\sigma_{x a}$ (these inputs are assumed to arrive from parts of the brain not directly included in our model). However, the inputs in real networks are represented not by continuous signals but by spike trains. Let us assume that neurons of a population $a$ receive $K_{a e}^{x}$ excitatory and $K_{a i}^{x}$ inhibitory external inputs, which have the synaptic weights equal to $j_{a e}^{x}, j_{a i}^{x}$ and the presynaptic firing rates equal to $r_{a e}^{x}, r_{a i}^{x}$, respectively. We want to check whether it is possible to select reasonable values for the aforementioned parameters of the external inputs that provide the values of $h_{x a}$ and $\sigma_{x a}$ that we used for the model. One of the appropriate combinations of these parameters for model 1 is the following: $K_{e e}^{x}=K_{i e}^{x}=4000, K_{e i}^{x}=K_{i i}^{x}=1000, r_{e e}^{x}=$ $r_{i e}^{x}=2.5 \mathrm{~Hz}, r_{e i}^{x}=r_{i i}^{x}=5 \mathrm{~Hz}, j_{e e}^{x}=0.44 \mathrm{mV}, j_{e i}^{x}=1.57 \mathrm{mV}$, $j_{i e}^{x}=0.62 \mathrm{mV}, j_{i i}^{x}=1.60 \mathrm{mV}$. For model 2, the appropriate weights are $j_{e e}^{x}=0.44 \mathrm{mV}, j_{e i}^{x}=1.57 \mathrm{mV}, j_{i e}^{x}=0.67 \mathrm{mV}$, $j_{i i}^{x}=1.57 \mathrm{mV}$. We suggest that these parameters fall into a physiologically reasonable range of values.

As we use linearized versions of the gain functions in our model, the firing rates could, in principle, become negative. However, we fixed the steady state and the amplitude of the induced oscillations in such a way that this did not happen. Specifically, the minimal firing rate for the excitatory and inhibitory population during the oscillations $\left(\tilde{r}_{a 0}-\left|r_{a}^{A}\right|\right)$ was equal to 17.1 and $34.0 \mathrm{~Hz}$ in model 1 , and 26.9 and $66.0 \mathrm{~Hz}$ in model 2.

The values of $\mathrm{CV}$ (numerically obtained from the simulations of individual LIF neurons) at the unforced steady state $S_{0}$ were equal to 1.1 for the excitatory neurons and 0.9 for the inhibitory neurons, which corresponds to Poisson-like spiking that is usually observed experimentally for most of the cortical neurons. Values of $\mathrm{CV}$ under external forcing are not fully tractable, as the spike trains are partially modulated by oscillations. However, we can consider a system that has the equilibrium state $\tilde{S}_{0}$ in the presence of purely asynchronous input with appropriately increased mean. For the parameters of model 1 , the $\mathrm{CV}$ in such system was equal to 1.1 for the excitatory neurons and 0.9 for the inhibitory neurons; for the parameters of model 2, the CV values of the excitatory and inhibitory neurons were equal to 1.0 and 0.8 , respectively. From this, we can conclude that the equilibrium shift produced by the external forcing did not move the system out of the physiologically plausible region of asynchronous firing.

The difference between the spiking threshold and the timeaveraged forced equilibrium voltage for the excitatory and inhibitory neurons $\left(V_{a}^{\text {th }}-\tilde{V}_{a 0}\right)$ was equal to 19.3 and 13.7 $\mathrm{mV}$ in model 1 and to 16.7 and $9.6 \mathrm{mV}$ in model 2. These values are large compared to the amplitudes $\left|V_{a}^{A}\right|$ of the forced voltage oscillations $(2.37 \mathrm{mV}$ for the excitatory neurons and $0.60 \mathrm{mV}$ for the inhibitory neurons). Consequently, even during peaks of oscillations, the neurons are well below the spiking threshold, so spiking is driven by random fluctuations of the input and not by oscillations themselves. In summary, the system demonstrates hallmarks of the sparsely synchronous regime characterized by moderate periodic firing rate modulation and irregular fluctuation-driven spiking of individual neurons, which is believed to be typical for oscillatory cortical networks.

\section{APPENDIX B: ANALYSIS OF THE MODELS}

\section{Fixed points of the unforced system}

We now derive analytical expressions that describe fixed points of our model without the periodic forcing. Let us first reduce the system (6) to two algebraic equations with the excitatory rate $r_{e}$ and the NMDA current in the excitatory population $I_{\mathrm{NMDA}}$ as the variables. In the main text, we used the curves resulting from this derivation and we demonstrated that plotting these curves on the $\left(r_{e}, I_{\mathrm{NMDA} e}\right)$ phase plane provides a useful geometrical intuition about existence of equilibria (which are defined by intersections of these curves), as well as about stability of these equilibria.

Let us define the functions $\bar{V}_{e}\left(r_{e}\right)$ and $\bar{V}_{i}\left(r_{i}\right)$ in such a way that, for an input $u_{a}(a=e, i)$, the following identity is true:

$$
\bar{V}_{a}\left(r_{a}^{s s}\left(u_{a}\right)\right)=V_{a}^{s s}\left(u_{a}\right),
$$

i.e., if the input $u_{a}$ moves the firing rate towards some value $r_{a}$, then the same input moves the membrane voltage towards $\bar{V}_{a}\left(r_{a}\right)$. From the explicit linear expressions for $r_{a}^{s s}, V_{a}^{s s}$ [given by (7)], it follows that

$$
\bar{V}_{a}\left(r_{a}\right)=V_{a 0}+\frac{c_{V a}}{c_{r a}}\left(r_{a}-r_{a 0}\right) .
$$

Next, let us find the state $z_{F}^{*} \equiv\left(r_{e}^{*}, r_{i}^{*}, V_{e}^{*}, V_{i}^{*}\right)$ of the fast subsystem $z_{F} \equiv\left(r_{e}, r_{i}, V_{e}, V_{i}\right)$, towards which it converges at the fast time scale for a given state of the slow subsystem $z_{S} \equiv\left(I_{\mathrm{NMDA} e}, I_{\mathrm{NMDA} i}\right)$. By considering the first two equations of (6) (that govern the dynamics of the fast subsystem) and setting the time derivatives in the left-hand sides to zero, we get

$$
\begin{aligned}
r_{a}^{*}\left(I_{\mathrm{NMDA} e}, I_{\mathrm{NMDA} i}\right) & =P_{r a}+Q_{r a}^{e} I_{\mathrm{NMDA} e}+Q_{r a}^{i} I_{\mathrm{NMDA} i} \\
V_{a}^{*}\left(I_{\mathrm{NMDA} e}, I_{\mathrm{NMDA} i}\right) & =\bar{V}_{a}\left(r_{a}^{*}\left(I_{\mathrm{NMDA} e}, I_{\mathrm{NMDA} i}\right)\right),
\end{aligned}
$$

where

$$
\begin{aligned}
Q_{r e}^{e} & =\frac{c_{r e}\left(1+c_{r i} J_{i i}\right)}{g_{m e} Q} \\
Q_{r e}^{i} & =\frac{-c_{r e} c_{r i} J_{e i}}{g_{m i} Q} \\
Q_{r i}^{e} & =\frac{c_{r e} c_{r i} J_{i e}^{\mathrm{AMPA}}}{g_{m e} Q} \\
Q_{r i}^{i} & =\frac{c_{r i}\left(1-c_{r e} J_{e e}^{\mathrm{AMPA}}\right)}{g_{m i} Q}
\end{aligned}
$$




$$
\begin{aligned}
Q & =\left(1-c_{r e} J_{e e}^{\mathrm{AMPA}}\right)\left(1+c_{r i} J_{i i}\right)+c_{r e} c_{r i} J_{e i} J_{i e}^{\mathrm{AMPA}} \\
P_{r e} & =r_{e 0}-Q_{r e}^{e} I_{\mathrm{NMDAe} 0}-Q_{r e}^{i} I_{\mathrm{NMDAi} 0} \\
P_{r i} & =r_{i 0}-Q_{r i}^{e} I_{\mathrm{NMDAe} 0}-Q_{r i}^{i} I_{\mathrm{NMDAi} 0} .
\end{aligned}
$$

Now we can conclude that the steady state firing rates and NMDA currents should satisfy

$$
\begin{aligned}
I_{\mathrm{NMDA} a} & =I_{\mathrm{NMDA} a}^{s s}\left(r_{e}, \bar{V}_{a}\left(r_{a}\right)\right) \\
r_{a} & =r_{a}^{*}\left(I_{\mathrm{NMDA} e}, I_{\mathrm{NMDA} i}\right) .
\end{aligned}
$$

Using (B3), let us express $r_{i}$ and $I_{\mathrm{NMDA} i}$ from (B5) as functions of $r_{e}$ and $I_{\mathrm{NMDA} e}$ :

$$
\begin{aligned}
\bar{r}_{i}\left(r_{e}, I_{\mathrm{NMDA} e}\right)= & \frac{Q_{r i}^{i}}{Q_{r e}^{i}}\left[r_{e}-\left(P_{r e}+Q_{r e}^{e} I_{\mathrm{NMDA} e}\right)\right] \\
& +\left(P_{r i}+Q_{r i}^{e} I_{\mathrm{NMDA} e}\right) \\
\bar{I}_{\mathrm{NMDA} i}\left(r_{e}, I_{\mathrm{NMDA} e}\right)= & I_{\mathrm{NMDA} i}^{s s}\left(r_{e}, \bar{V}_{i}\left(\bar{r}_{i}\left(r_{e}, I_{\mathrm{NMDA} e}\right)\right)\right) .
\end{aligned}
$$

Finally, in order to obtain self-consistent equations for $r_{e}$ and $I_{\mathrm{NMDA} e}$, let us put (B6) back into (B5):

$$
\begin{aligned}
r_{e} & =P_{r e}+Q_{r e}^{e} I_{\mathrm{NMDA} e}+Q_{r e}^{i} \bar{I}_{\mathrm{NMDA} i}\left(r_{e}, I_{\mathrm{NMDA} e}\right) \\
I_{\mathrm{NMDA} e} & =I_{\mathrm{NMDA} e}^{s s}\left(r_{e}, \bar{V}_{e}\left(r_{e}\right)\right) .
\end{aligned}
$$

As we mentioned earlier, the first and the second equations of (B7) define two curves on the $\left(r_{e}, I_{\mathrm{NMDA} e}\right)$ plane, intersections of which correspond to the fixed points of the system (6). In the main text, we refer to these curves as the $r_{e}$ curve and the $I_{\mathrm{NMDA}}$ curve, respectively. We should note that these curves are not nullclines, although they intersect at the fixed points of the system.

\section{Oscillation-induced shift of the time-averaged equilibrium}

Here we derive the expression for the dynamics of the timeaveraged slow subsystem. To do this, we should analytically estimate the integral in (15) that links the dynamics of the original and the time-averaged slow subsystem in the presence

of periodic forcing. First, let us expand $g_{\mathrm{NMDA}}$ about $V_{a}^{*}$ :

$$
\begin{aligned}
g_{\mathrm{NMDA}}\left(V_{a}\right) & =\sum_{k=0}^{\infty} \frac{1}{k !} g_{a k}\left(V_{a}-V_{a}^{*}\right)^{k} \\
g_{a k} & =\left.\frac{d g_{\mathrm{NMDA}}\left(V_{a}\right)}{d V_{a}}\right|_{V_{a}^{*}} .
\end{aligned}
$$

Given the expansion (B8), we get

$$
\begin{aligned}
g_{\mathrm{NMDA}} & \left(V_{a}^{*}+\left|V_{a}^{A}\right| \cos \left(\omega t+\psi_{V a}\right)\right)\left(r_{e}^{*}+\left|r_{e}^{A}\right| \cos \left(\omega t+\psi_{r a}\right)\right) \\
= & r_{e}^{*} g_{\mathrm{NMDA}}\left(V_{a}^{*}\right)+\left|r_{e}^{A}\right| g_{\mathrm{NMDA}}\left(V_{a}^{*}\right) \cos \left(\omega t+\psi_{r a}\right)+r_{e}^{*}\left|V_{a}^{A}\right| g_{\mathrm{NMDA}}\left(V_{a}^{*}\right) \cos \left(\omega t+\psi_{V a}\right) \\
& +\left|r_{e}^{A}\right|\left|V_{a}^{A}\right| g_{\mathrm{NMDA}}^{\prime}\left(V_{a}^{*}\right) \cos \left(\omega t+\psi_{V a}\right) \cos \left(\omega t+\psi_{r a}\right) \\
& +\frac{1}{2} r_{e}^{*}\left|V_{a}^{A}\right|^{2} g^{\prime \prime}{ }_{\mathrm{NMDA}}\left(V_{a}^{*}\right) \cos ^{2}\left(\omega t+\psi_{V a}\right)+o\left(\left|r_{e}^{A}\right|^{2}\right) \\
= & r_{e}^{*} g_{\mathrm{NMDA}}\left(V_{a}^{*}\right)+\left|r_{e}^{A}\right| g_{\mathrm{NMDA}}\left(V_{a}^{*}\right) \cos \left(\omega t+\psi_{r a}\right)+r_{e}^{*}\left|V_{a}^{A}\right| g_{\mathrm{NMDA}}\left(V_{a}^{*}\right) \cos \left(\omega t+\psi_{V a}\right) \\
& +\frac{1}{2}\left|r_{e}^{A}\right|\left|V_{a}^{A}\right| g_{\mathrm{NMDA}}^{\prime}\left(V_{a}^{*}\right)\left(\cos \left(2 \omega t+\psi_{V a}+\psi_{r a}\right)+\cos \left(\psi_{V a}-\psi_{r a}\right)\right) \\
& +\frac{1}{4} r_{e}^{*}\left|V_{a}^{A}\right|^{2} g_{\mathrm{NMDA}}^{\prime \prime}\left(V_{a}^{*}\right)\left(\cos \left(2 \omega t+2 \psi_{V a}\right)+1\right)+o\left(\left|r_{e}^{A}\right|^{2}\right),
\end{aligned}
$$

where we take into account that $\left|r_{e}^{A}\right|,\left|r_{i}^{A}\right|,\left|V_{e}^{A}\right|,\left|V_{i}^{A}\right|$ have the same order of magnitude.

The explicit expression for $G_{a}^{\text {osc }}$ [which is integrated in (15)] is

$$
G_{a}^{\mathrm{osc}}\left(I_{\mathrm{NMDA} e}, I_{\mathrm{NMDA} i}, t\right)=\frac{1}{\tau_{\mathrm{NMDA}}}\left(J_{a e}^{\mathrm{NMDA}} g_{\mathrm{NMDA}}\left(V_{a}^{*}+\left|V_{a}^{A}\right| \cos \left(\omega t+\psi_{V a}\right)\right)\left(r_{e}^{*}+\left|r_{e}^{A}\right| \cos \left(\omega t+\psi_{r a}\right)\right)-I_{\mathrm{NMDA} a}\right) .
$$

Now we put (B9) into (B10) and omit the $o\left(\left|r_{e}^{A}\right|^{2}\right)$ terms, after which we can explicitly calculate the integral in (15). As we integrate over the period of oscillations, all the timedependent terms vanish, which gives us the final result (16).

\section{APPENDIX C: DESCRIPTION OF THE BIFURCATION DIAGRAMS}

In this section, we provide a complete description of the bifurcation diagrams depicted in Fig. 8. We successively go from small to large values of $J_{i e}^{\mathrm{NMDA}}$ [referred to by numbers from 1 to 9 in Fig. 8(a)] and describe the events that occur as $J_{i e}^{\mathrm{NMDA}}$ is varied.

The values 1 and 2 represent the case in which only one saddle-node bifurcation is possible. It corresponds to crossing of the line AB by lines 1 and 2 in Fig. 8(a) and by diagrams 1 and 2 in Figs. 8(c) and 8(d). Model 1 considered in the paper belongs to this case.

For the value 3 , in addition to the aforementioned saddlenode bifurcation, one can observe the cusp which is denoted by the point $\mathrm{C}$ in Figs. 8(a)-8(d). For larger values of $J_{i e}^{\mathrm{NMDA}}$, two saddle-node lines $\mathrm{CB}$ and $\mathrm{CD}$ grow from the cusp point C. According to this, diagram 4 in Fig. 8(c) contains three 
saddle-node bifurcation points. Let us describe the sequence of events that occur as $J_{i e}^{\mathrm{AMPA}}$ decreases from the maximal value to zero.

(1) For large $J_{i e}^{\mathrm{AMPA}}$, diagram 4 has two branches: the lower one contains stable nodes, and the upper one contains saddle points.

(2) As $J_{i e}^{\mathrm{AMPA}}$ decreases, diagram 4 crosses the saddlenode bifurcation line $\mathrm{BC}$, and two additional middle branches appear. The lower-middle branch contains saddle points; the upper-middle branch contains stable nodes.

(3) As $J_{i e}^{\mathrm{AMPA}}$ further decreases, line 4 in Fig. 8(a) crosses the green line, and the fixed points lying on the upper-middle branch of diagram 4 become stable foci.

(4) After this, line 4 in Fig. 8(a) crosses the Hopf bifurcation line $\mathrm{CG}$, and the fixed points lying on the upper-middle branch of diagram 4 become unstable foci (via supercritical Hopf bifurcation).

(5) After the Hopf bifurcation, alongside with the destabilization of the fixed point, a stable limit cycle appears. An additional analysis demonstrated that this cycle exists only in the narrow range of $J_{i e}^{\mathrm{AMPA}}$ values just below the bifurcation point. For even smaller $J_{i e}^{\mathrm{AMPA}}$, the limit cycle disappears via homoclinic bifurcation.

(6) Eventually, diagram 4 crosses the saddle-node bifurcation line $\mathrm{AB}$, and the lower stable branch and the lowermiddle unstable branch merge and disappear. For smaller $J_{i e}^{\mathrm{AMPA}}$ values, there are no stable fixed points in the system.

(7) Finally, line 4 in Fig. 8(a) crosses the blue line that marks the border of instability of the unforced system. At this point, diagram 4 in Figs. 8(c) and 8(d) changes its color from blue to gray.

Diagrams 5 and 6 differ from diagram 4 in that their lower branch contains stable foci for large $J_{i e}^{\mathrm{AMPA}}$ values, instead of stable nodes.

The important difference of diagram 6 from diagram 5 is that the Hopf bifurcation occurs for diagram 6 for a smaller value of $J_{i e}^{\mathrm{AMPA}}$ than the saddle-node bifurcation [this is true for all values of $J_{i e}^{\mathrm{NMDA}}$ that lie between the points $\mathrm{E}$ and B in the Fig. 8(a)]. As we move along diagram 6 from the right to the left, the lower two branches merge and disappear via the saddle-node bifurcation, but for somewhat smaller $J_{i e}^{\mathrm{AMPA}}$ values the system would not diverge, but jump to the uppermiddle branch, as this branch is stable.

The $J_{i e}^{\mathrm{NMDA}}$ value 7 corresponds to the cusp point B. In diagram 7 , the unstable lower-middle branch disappears, and the lower stable branch and the upper-middle stable branch merge. The diagrams corresponding to higher $J_{i e}^{\mathrm{NMDA}}$ (such as 8 and 9) contain a single stable branch. In diagram 8 , some of the stable points are nodes, while in diagram 9 all the stable points are foci.

We should describe certain issues related to the production of Fig. 8. The saddle-node bifurcation, the Hopf bifurcation, and the node-to-focus transition occur very close to each other in the parameter plane [see the upper part of Fig. 8(b)]. Thus, the continuation procedure is challenging in this case, and we used a "bruteforce" approach: we calculated the relevant quantities [eigenvalues for Figs. 8(a) and 8(b) and fixed-point conditions for Figs. 8(c) and 8(d)] in the nodes of a thin grid, and then plotted the bifurcations lines as boundaries between regions with different properties. This resulted in small artifactual discontinuities in the diagram branches near the saddle-node bifurcation points [near the turning points of the blue lines in Figs. 8(c) and 8(d)], so we connected the ending points of the adjacent branch segments by straight lines. We assumed that the saddle-node bifurcation points lie close to the middle of these lines, so we connected the corresponding midpoints of multiple branches [we calculated more branches than it is shown in Figs. 8(c) and 8(d)] to produce the saddle-node bifurcation line [the black $A B C D$ line in Figs. 8(c) and 8(d)]. The resulting ABCD line was smoothed for illustrative purposes. Similarly, the thick red line in Figs. 8(c) and 8(d) was drawn through the Hopf bifurcation points located at multiple branches and then smoothed for illustrative purposes. This procedure failed to produce the segment of the red line near the point $\mathrm{C}$ (where the Hopf and the saddle-node bifurcation lines approach each other and finally intersect), so we drew this segment manually [thin red line segment in the Figs. 8(c) and 8(d)]. The aforementioned manipulations (reconnection of branches, approximation of the saddle-node points, smoothing, and manual segment addition) were performed for better presentation of the results only and had no effect on the conclusions.
[1] X. J. Wang, Physiol. Rev. 90, 1195 (2010).

[2] P. Fries, J. H. Reynolds, A. E. Rorie, and R. Desimone, Science 291, 1560 (2001).

[3] E. T. Rolls and A. Treves, Prog. Neurobiol. 95, 448 (2011).

[4] M. Vinck, T. Womelsdorf, and P. Fries, in Principles of Neural Coding, edited by R. Quian Quiroga and S. Panzeri (CRC, Boca Raton, FL, 2013), p. 449.

[5] T. Akam and D. M. Kullmann, Nat. Rev. Neurosci. 15, 111 (2014).

[6] P. Fries, Trends Cogn. Sci. 9, 474 (2005).

[7] M. Dipoppa and B. S. Gutkin, PNAS 110, 12828 (2013).

[8] J. S. Brittain, A. Sharott, and P. Brown, Eur. J. Neurosci. 39, 1951 (2014).

[9] H. Schmidt, D. Avitabile, E. Montbrio, and A. Roxin, PLoS Comput. Biol. 14, e1006430 (2018).

[10] T. Akam and D. M. Kullmann, Neuron 67, 308 (2010).

[11] N. Brunel and X. J. Wang, J. Neurophysiol. 90, 415 (2003).
[12] A. K. Roopun, M. A. Kramer, L. M. Carracedo, M. Kaiser, C. H. Davies, R. D. Traub, N. J. Kopell, and M. A. Whittington, Front. Cell. Neurosci. 2, 1 (2008).

[13] S. Vijayan and N. J. Kopell, PNAS 109, 18553 (2012).

[14] E. Salinas and T. J. Sejnowski, J. Neurosci. 20, 6193 (2000).

[15] S. Voronenko and B. Lindner, New J. Phys. 19, 033038 (2017).

[16] S. H. Strogatz, Nonlinear Dynamics and Chaos; Steven $H$. Strogatz with Applications to Physics, Biology, Chemistry, and Engineering (Westview, Cambridge, MA, 2000).

[17] R. T. Canolty, K. Ganguly, and J. M. Carmena, PLoS Comput. Biol. 8, e1002809 (2012).

[18] M. Lundqvist, J. Rose, P. Herman, S. L. Brincat, T. J. Buschman, and E. K. Miller, Neuron 90, 152 (2016).

[19] M. Shafi, Y. Zhou, J. Quintana, C. Chow, J. Fuster, and M. Bodner, Neuroscience 146, 1082 (2007).

[20] M. V. Tsodyks and H. Markram, PNAS 94, 719 (1997). 